\title{
Detecting and Estimating Signals over Noisy and Unreliable Synapses: Information-Theoretic Analysis
}

\author{
Amit Manwani \\ Christof Koch \\ Computation and Neural Systems, California Institute of Technology, Pasadena, CA \\ 91125, U.S.A
}

The temporal precision with which neurons respond to synaptic inputs has a direct bearing on the nature of the neural code. A characterization of the neuronal noise sources associated with different sub-cellular components (synapse, dendrite, soma, axon, and so on) is needed to understand the relationship between noise and information transfer. Here we study the effect of the unreliable, probabilistic nature of synaptic transmission on information transfer in the absence of interaction among presynaptic inputs. We derive theoretical lower bounds on the capacity of a simple model of a cortical synapse under two different paradigms. In signal estimation, the signal is assumed to be encoded in the mean firing rate of the presynaptic neuron, and the objective is to estimate the continuous input signal from the postsynaptic voltage. In signal detection, the input is binary, and the presence or absence of a presynaptic action potential is to be detected from the postsynaptic voltage. The efficacy of information transfer in synaptic transmission is characterized by deriving optimal strategies under these two paradigms. On the basis of parameter values derived from neocortex, we find that single cortical synapses cannot transmit information reliably, but redundancy obtained using a small number of multiple synapses leads to a significant improvement in the information capacity of synaptic transmission.

\section{Introduction}

Understanding the strategies that brains use to represent and process information received through the senses and to make crucial behavioral decisions has been a long-standing goal in neuroscience. Researchers continue to remain divided on the nature of the neural code, though a lot of recent effort in theoretical neuroscience has focused on deciphering the code. A common notion in neuroscience is that spike trains are stochastic and the average firing rate of a neuron constitutes the primary variable relating neuronal response to sensory experience (Adrian, 1932; Barlow, 1972). This view is supported by the demonstration of a quantitative relationship between the average firing rate of single cortical neurons and the performance of the 
animal in behavioral tasks (Werner \& Mountcastle, 1963; Newsome, Britten, \& Movshon, 1989; Zohary, Hillman, \& Hochstein, 1990; Britten, Shadlen, Newsome, \& Movshon, 1992).

On the other hand, recent findings indicate that spike timing can be highly precise, and as a result, neurons can potentially employ the fine temporal structure of interspike intervals to convey more information than a mean rate code (Rieke, Warland, van Steveninck, \& Bialek, 1997). The relative timing of spikes across a population of neurons also appears to be relevant for coding in certain cases (Perkel \& Bullock, 1968; Abeles, 1990; Bialek, Rieke, van Steveninck, \& Warland, 1991; Bialek \& Rieke, 1992; Richmond \& Optican, 1992; Rieke et al., 1997). It is unclear which, if there is one in particular, is the universal coding strategy that brains use. However, a quantitative understanding of neuronal noise sources and their influence on reliability and precision of neuronal signaling will determine the efficacy of neurons in transmitting information and the constraints under which neuronal codes must operate.

Tools from statistical estimation theory (Poor, 1994) have recently been used to quantify the ability of neurons to transmit information through their spike outputs (Bialek et al., 1991; Bialek \& Rieke, 1992; Gabbiani, 1996; Rieke et al., 1997). These techniques have been successfully applied to study the coding of random stimuli in peripheral sensory neurons in various neural systems. Most of these approaches treat the neuron as a black box, ignoring the details of neural information processing, and estimate neuronal information capacity by statistical analyses of the associated empirical inputoutput records. However, it is well known that neurons receive inputs at their synapses; synaptic inputs are integrated in the dendrites and delivered to the spike initiation zone, which generates the output spike train and transmits it via the axon to the neuron's postsynaptic targets. The specific nature of information processing in the nervous system is determined by the details of this neural hardware. Much knowledge has accumulated about the manner in which signals are processed and transformed at various stages in single neurons through extensive experimental and theoretical efforts (Koch, 1999). However, the relationship between single-neuron biophysics and neural information processing has not been unraveled in a systematic and principled manner. We believe that a detailed understanding of the different neuronal components can provide deeper insight into the role of neurons as communication devices. Thus, a reductionist approach of applying experimental and theoretical tools to individual neuronal components will reveal aspects of the neural code that cannot be uncovered using conventional black box approaches.

Since noise has a direct bearing on the nature of coding and limits the capacity of information processing systems, an essential step in determining the functional significance of the different processing stages in neuronal information transfer (synapse, dendritic tree, axon, and so on) involves the characterization of the intrinsic biophysical noise they contribute. We have 
used this approach to study the effect of different membrane noise sources on information processing in dendritic cable models of cortical neurons in the presence of low densities of voltage-gated ion channels (Manwani \& Koch, 1999a, 1999b, 1999c; Steinmetz, Manwani, London, Segev, \& Koch, 2000). Here we focus on a critical component of neural processing, the synapse. Using the known biophysical details about cortical synapses, we derive a simple mathematical model of a cortical synapse. Our model ignores the ubiquitous use-dependent plasticity of cortical synapses and thus represents a simplified picture of synaptic transmission. It can be seen as a first step in modeling and quantifying the effects of synapses on information processing. We compute the information-theoretical capacity of this synaptic model under two explicit coding paradigms: signal estimation and signal detection.

In the signal estimation paradigm, the input is a random continuous signal encoded in the mean firing rate of a presynaptic neuron. Using tools from statistical estimation theory, we derive the optimal filter (in the sense of least-mean square error), which reconstructs the input from the postsynaptic voltage. In the signal detection paradigm, the input signal is encoded in an all-or-none format (the presence or absence of a presynaptic spike). We derive the optimal detector (in the sense of minimum probability of error) of presynaptic action potentials from the postsynaptic voltage. The signal detection method is similar to the yes-no decision paradigm used in psychophysics. We use the performance of the optimal estimator and optimal detector to characterize the synaptic efficacy for these two tasks and to derive bounds on the information capacity of cortical synapses.

The strength of our approach lies in the combination of techniques from different scientific fields - single-neuron biophysics (Hille, 1992; Koch, 1999), membrane noise analysis (DeFelice, 1981; Traynelis \& Jaramillo, 1998; White, Rubinstein, \& Kay, 2000), cable theory, estimation theory (Poor, 1994), and information theory (Cover \& Thomas, 1991) - and in their application to the question of neural coding from a novel perspective. Ultimately our goal is to answer questions like, Is the length of the apical dendrite of a neocortical pyramidal cell limited by considerations of signal-to-noise? What influences the noise level in the dendritic tree of a real neuron endowed with voltagedependent channels? How accurately can the time course of an synaptic signal be reconstructed from the voltage at the spike initiation zone? and What is the channel capacity of an unreliable synapse onto a spine? A brief account of this work has appeared previously (Manwani \& Koch, 1998).

\section{The Stochastic Nature of Synaptic Transmission}

Our understanding of synaptic transmission is based on the seminal work of Katz and his collaborators (Katz, 1969). Their central findings were that the release of neurotransmitter occurs in quanta through vesicles residing in the presynaptic terminal. Each vesicle is released independently of the others in 
a probabilistic manner, and the postsynaptic responses to vesicular release add linearly. Their theory was developed using experiments at the frog neuromuscular junction, but the stochastic and quantal nature of synaptic transmission has been found to be generally valid for central synapses as well. The probability of vesicle release at a single active zone of the frog neuromuscular junction was found to be quite low. However, this junction contains a large number of release sites (on the order of 1000), and as a result, the neuromuscular synapse is highly reliable. Thus, a presynaptic action potential invariably gives rise to a muscular response.

While central synapses share many principles in common with neuromuscular junctions, there are crucial differences between them as well (Redman, 1990; Stevens, 1993). Synaptic boutons in central synapses contain only one or a few active release zones as opposed to the 1,000 or more found at the neuromuscular junction. Thus, in response to an action potential in the presynaptic terminal, at most one vesicle is released (Korn \& Faber, 1991, 1993; Stevens \& Wang, 1995). Moreover, in vitro studies in vertebrate and invertebrate systems have revealed that the probability of vesicle release (referred to as $p$ ) is generally low (Korn, Faber, \& Triller, 1986; Laurent \& Sivaramakrishnan, 1992; Bekkers \& Stevens, 1994, 1995; Stevens \& Wang, 1994). The probabilistic release of vesicles is believed to be the dominant factor responsible for the unreliability of synaptic transmission in cortical and hippocampal neurons (Allen \& Stevens, 1994). The origin of the random nature of release is not yet fully understood, though it is known that the precise three-dimensional spatial relationship between calcium channels in the presynaptic membrane and the location of the vesicles determines release dynamics.

The probability of synaptic release is not constant; it depends on whether the last presynaptic action potential led to a vesicle release and the exact timing of the previous presynaptic spikes. Depending on the sequence of presynaptic action potentials, $p$ can either decrease or increase over a variety of timescales, from tens of milliseconds to seconds, minutes, and longer. This history-dependent plasticity in the state of synaptic efficacy is believed to be one of the substrates of learning and memory in biological neural networks (Abbott, Varela, Sen, \& Nelson, 1997). $p$ can also vary across synapses (Dobrunz \& Stevens, 1997; Murthy, Sejnowski, \& Stevens, 1997) and synaptic (Stratford, Tarczy-Hornoch, Martin, Bannister, \& Jack, 1996) types impinging onto a single neuron.

The unreliability of synaptic transmission in cortical neurons is compounded by the variability in the amplitude of the postsynaptic response following the successful release of a single vesicle (Bekkers, Richerson, \& Stevens, 1990; Edwards, Konnerth, \& Sakmann, 1990; Mason, Nicoll, \& Stratford, 1991). This response variability occurs due to factors like variation in vesicle size and therefore in the number of neurotransmitter molecules, the number of available postsynaptic receptors, and so on. The trial-to-trial variability in postsynaptic amplitude can be quite large; in some cases, the 
variance in the size of the excitatory postsynaptic potential (EPSP) is as large as the mean (Bekkers et al., 1990; Larkman, Stratford, \& Jack, 1991; Mason et al., 1991). A note of caution is in order here: a majority of studies of central synapses have been carried out in culture or slice preparations that lack many of the neuromodulators present in vivo. This might significantly affect synaptic release properties. Only after a careful measurement of synaptic properties in in vivo experiments will the true picture of synaptic transmission in real brains emerge.

In summary, central synapses appear to be highly unreliable, binary connections. This unreliability is several orders of magnitude higher than that observed in engineering systems (Koch, 1999). Theoretically, the nervous system can combat the unreliability of its components by making use of anatomical redundancy with multiple synapses (Moore \& Shannon, 1956; von Neumann, 1956). However, cortical axons typically make only a few synaptic contacts onto other cortical target neurons (Sorra \& Harris, 1993). Thus, the reliability of synaptic transmission must have a profound influence on the manner in which signals are encoded and transmitted (Abbott et al., 1997; Lisman, 1997; Tsodyks \& Markram, 1997; Zador, 1998) and the ways in which computation is performed in the brain (Stevens, 1994).

An important debate that rages on is whether synaptic unreliability is a "bug" or a "feature" (Koch, 1999). In other words, do biophysical constraints limit the potential reliability of cortical synapses (for instance, the need to pack on the order of 1 billion synapses into 1 cubic millimeter of cortex might dictate the small size of synapses) or are there any computational advantages to this unreliability? If reliable synapses that are as small and compact as hippocampal synapses are found experimentally, it would certainly give credence to the hypothesis that synaptic unreliability is probably a design feature used in the brain. One possible computational advantage to having unreliable synapses is for reasons of plasticity. It has been argued that the release probability can be modified more easily over a larger dynamic range than the number of release sites, or the size of the postsynaptic response (Smetters \& Zador, 1996; Zador \& Dobrunz, 1997). Thus, synaptic unreliability could lead to an increase in the bandwidth of modulation of the postsynaptic response.

It has also been suggested (Burnod \& Korn, 1989) that the variability at central synapses plays the same role as the "temperature" parameter $T$ in stochastic models of neuronal networks (Ackley, Hinton, \& Sejnowski, 1985). Just as the presence of noise in large artificial neural networks prevents them from getting stuck in local minima, synaptic unreliability and variability might help brains to learn and generalize better.

\section{Channel Model of Synaptic Transmission}

Armed with the above knowledge about the physiology of central synapses, we abstract a synapse by a mathematical model that accounts for probabilis- 


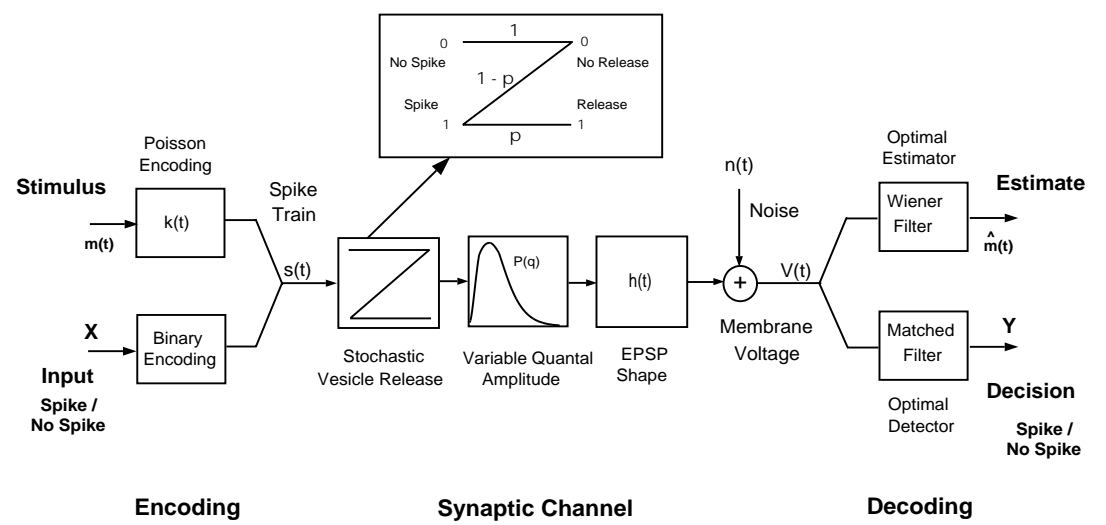

Figure 1: Schematic block diagram of a synapse under the signal estimation and detection paradigms. A cortical synapse is modeled as a binary channel followed by a filter $h(t)$. The amplitude of the filter is a random variable $q$ with probability density $P(q)=a(a q)^{J-1} \exp (-a q) /(J-1)$ ! The binary channel (inset: $p=$ Prob [vesicle release], $1-p=$ Prob [failure]) models probabilistic vesicle release, and the random variable $q$ models the variability in the size of the postsynaptic response of a single quantum observed in central synapses. The EPSP is approximated by an alpha function $h(t)=h_{\text {peak }} t / t_{\text {peak }} \exp (1-$ $\left.t / t_{\text {peak }}\right) . n(t)$ denotes additive postsynaptic voltage noise, which is assumed to be gaussian and white (over a bandwidth $B_{\mathrm{n}}$ ). In signal estimation, the objective is to estimate optimally a continuous input $m(t)$ from the postsynaptic membrane voltage $V(t)$. This estimate is denoted by $\hat{m}(t)$. The presynaptic spike train $s(t)$ is assumed to be a Poisson process with firing rate modulated by the random input $m(t)$. Performance of the optimal linear estimator (Wiener filter) is used to quantify performance in the estimation task. In signal detection, the objective is to detect optimally the presence of a single presynaptic action potential on the basis of $V(t)$. Thus, the input $X$ and the decision $Y$ are binary variables. Performance of the optimal detector (matched filter) quantifies performance in signal detection.

tic vesicle release and the variability in response amplitude. Using the model as a starting point, we will derive its information-theoretic channel equivalents under the signal estimation and signal detection tasks. The capacities of these channels will be used to quantify the efficacy of synaptic transmission under the two paradigms. (Appendix A lists the symbols used.)

We will assume that the synaptic bouton contains only one release site. Thus, the vesicle release process can be modeled as a binary channel (see the inset of Figure 1). The input to the channel is a binary variable that represents the presence or the absence of a presynaptic action potential, and its output is a binary variable representing the success or failure of release. The spontaneous release associated with cortical synapses is quite 
low, and so we shall assume that it is zero here (Zador, 1998). Our analysis, however, does not depend on this assumption. Let $p$ denote the probability of release due to a presynaptic spike. The unreliability of vesicle release is the dominant source of noise in the release process as $p$ can be as low as 0.1 for some hippocampal synapses (Hessler, Shirke, \& Malinow, 1993).

We model the postsynaptic response to the release of a single vesicle by a function $h(t)$, which corresponds to the EPSP waveform of a fast, voltageindependent AMPA-like synapse modeled as an alpha function (Rall, 1967),

$$
h(t)=h_{\text {peak }} \frac{t}{t_{\text {peak }}} \exp \left(1-\frac{t}{t_{\text {peak }}}\right),
$$

where $h_{\text {peak }}$ is the peak EPSP magnitude and $t_{\text {peak }}$ is the corresponding timeto-peak. We assume that the postsynaptic responses to a sequence of vesicle releases add linearly. We incorporate synaptic variability by multiplying the response $h(t)$ by a random variable $q$ drawn from a probability distribution $P(q)$, which can be measured empirically. Thus, $q$ models the trialto-trial variability in the amplitude of the postsynaptic responses observed for central neurons. Experimentally observed amplitude distributions are generally skewed toward higher amplitudes, though the experimental difficulties of measuring very small synaptic events probably also contributes to these findings (Bekkers \& Stevens, 1996). Here we model $P(q)$ by a gamma distribution,

$$
P(q)=a \frac{(a q)^{J-1}}{(J-1) !} \exp (-a q),
$$

where $J$ is the order of the distribution. However, it can be replaced by any one-sided probability density ${ }^{1}$ for the purpose of subsequent analysis. $a$ and $J$ together determine the spread of the distribution. $\bar{q}$ denotes the mean, and $\sigma_{\mathrm{q}}$ denotes the standard deviation of the quantal amplitude $q$ :

$$
\bar{q}=\frac{J}{a}, \quad \sigma_{\mathrm{q}}=\frac{\sqrt{J}}{a} .
$$

The coefficient of variance (denoted by $C V_{\mathrm{q}}$ ) is a measure of the amplitude variability and is given by

$$
C V_{\mathrm{q}}=\frac{\sigma_{\mathrm{q}}}{\bar{q}}=\frac{1}{\sqrt{J}}
$$

Thus, $J$ can be used to modify the variability of $q . J=1$ corresponds to an exponential distribution with the highest variability $\left(C V_{\mathrm{q}}=1\right)$; at the other extreme, $J=\infty$ corresponds to a delta function for which there is no variability in $q\left(C V_{\mathrm{q}}=0\right)$.

\footnotetext{
${ }^{1}$ Here we assume that $q$ is a nonnegative random variable; inhibitory synapses can be analyzed identically by reversing the sign of $h(t)$.
} 
In addition to these two sources of noise, we also assume that the postsynaptic membrane voltage is corrupted by additive, gaussian noise $n(t)$, which models the effect of other membrane noise sources like thermal noise, channel noise, background synaptic noise from other synapses, and so on (DeFelice, 1981). We denote the power spectral density of $n(t)$ by $S_{\mathrm{nn}}(f)$. We assume that $n(t)$ is band limited (over a bandwidth $B_{\mathrm{n}}$ ),

$$
\begin{aligned}
S_{\mathrm{nn}}(f) & =\frac{\sigma_{\mathrm{n}}^{2}}{2 B_{\mathrm{n}}} & & -B_{\mathrm{n}} \leq f \leq B_{\mathrm{n}} \\
& =0 & & \text { otherwise, }
\end{aligned}
$$

where $\sigma_{\mathrm{n}}^{2}$ is the variance of $n(t)$. We define a signal-to-noise ratio $S N R$ for the synapse as

$$
S N R=\frac{1}{S_{\mathrm{nn}}(f)} \int_{0}^{\infty} \mathrm{d} t h^{2}(t)=\frac{2 B_{\mathrm{n}}}{\sigma_{\mathrm{n}}^{2}} \int_{0}^{\infty} \mathrm{d} t h^{2}(t) .
$$

We have mathematically modeled a cortical synapse as a binary channel in cascade with a random amplitude filter and an additive gaussian noise source (see the schematic diagram in Figure 1). We ignore history-dependent effects (paired-pulse facilitation, vesicle depletion, calcium buffering, and so on) on synaptic transmission that endow central synapses with characteristics of sophisticated nonlinear filters (Markram \& Tsodyks, 1996; Abbott et al., 1997). This implies that the synaptic parameters in our model will be regarded as constants.

We derive theoretical lower bounds on the capacity of this simple model of synaptic transmission under the two representational paradigms: signal detection and signal estimation. In signal estimation, the signal is assumed to be encoded in the mean firing rate of the presynaptic neuron, and the objective is to estimate the continuous input signal from the postsynaptic voltage. In signal detection, the input is binary, and the presence or absence of a presynaptic action potential is to be detected from the postsynaptic voltage. The efficacy of information transfer in synaptic transmission is characterized by deriving optimal strategies under these two paradigms.

\section{Signal Estimation}

Let $m(t)$ be a zero-mean random stimulus presented to a presynaptic neuron and $s(t)$ the resulting spike train. $s(t)$ is modeled as a point process or as a sequence of delta functions,

$$
s(t)=\sum_{i} \delta\left(t-t_{i}\right)
$$

where $t_{i}$ denotes the time when the $i$ th spike occurs. We assume that $m(t)$ and $s(t)$ are (real-valued) jointly weak-sense stationary (WSS) processes with 
finite variances, $\left\langle m^{2}(t)\right\rangle=\sigma_{\mathrm{m}}^{2}<\infty,\left\langle|s(t)-\bar{\lambda}|^{2}\right\rangle<\infty$, where $\bar{\lambda}=\langle s(t)\rangle$ is the mean firing rate of the presynaptic neuron. In these equations, $\langle\cdot\rangle$ denotes an ensemble average over the joint stimulus and spike train distribution. The membrane voltage $V(t)$ at the postsynaptic site due to the transmission of the spike train $s(t)$ over the synapse is

$$
V(t)=\sum_{i} q_{i} W_{i} h\left(t-t_{i}\right)+n(t)
$$

where $q_{i}$ is the EPSP amplitude in response to the $i^{\text {th }}$ spike and $W_{i}$ is a binary variable representing vesicle release. Thus, $W_{i}=0$ implies that the $i^{\text {th }}$ presynaptic action potential did not lead to a vesicle release, whereas $W_{i}=$ 1 denotes a successful release. The mean voltage $\langle V(t)\rangle$ does not contain any information about the modulations of the input $m(t)$ and can be ignored. Let $v(t)$ refer to a zero-mean process obtained by subtracting the mean voltage from $V(t)$,

$$
v(t)=V(t)-\langle V(t)\rangle
$$

The objective in signal estimation is to find the optimal estimator of $m(t)$ from the postsynaptic voltage $v(t)$, where optimality is in the sense of least mean-square-error (MSE). In general, the optimal MSE estimator is nonlinear and complicated to treat analytically. Instead, we restrict ourselves to the analysis of the optimal linear estimator,

$$
\hat{m}(t)=g(t) \star v(t)
$$

where the $f \star g$ denotes the convolution between two functions $f$ and $g \cdot g(t)$ is the optimal linear filter that minimizes the MSE between the stimulus $m(t)$ and the estimate $\hat{m}(t)$,

$$
\mathcal{E}=\left\langle|m(t)-\hat{m}(t)|^{2}\right\rangle
$$

Using the orthogonality principle (Papoulis, 1991), $g(t)$ can be obtained by solving the equation,

$$
R_{\mathrm{vm}}(z)=\left(g \star R_{\mathrm{vv}}\right)(z)
$$

where $R_{\mathrm{vv}}(z)=\langle v(t) v(t+z)\rangle$ is the auto-correlation of the postsynaptic voltage and $R_{\mathrm{vm}}(z)=\langle v(t) m(t+z)\rangle$ is the cross-correlation between the stimulus and the membrane voltage. Taking the Fourier transform of both sides of equation 4.4,

$$
G(f)=\frac{S_{\mathrm{vm}}(f)}{S_{\mathrm{vv}}(f)},
$$




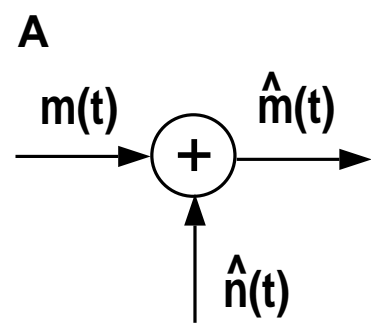

Estimation Channel

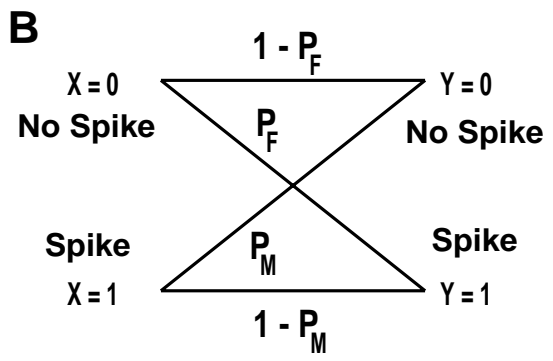

Detection Channel

Figure 2: Channel models of synaptic performance under signal estimation and detection. (A) Effective channel model of the synapse for the signal estimation paradigm. The random stimulus $m(t)$ is the continuous input to the channel, and $\hat{m}(t)$ is the best linear estimate of the input based on the postsynaptic voltage (see Figure 1). The effective reconstruction noise $\hat{n}(t)$ is the difference between the input and the estimate, $\hat{n}(t)=m(t)-\hat{m}(t)$. (B) Effective channel model of the synapse for the signal detection paradigm. $X$ and $Y$ are binary random variables corresponding to the input and the decision, respectively. The false alarm and the miss probabilities $\left(P_{\mathrm{F}}\right.$ and $P_{\mathrm{M}}$, respectively), which minimize the detection error $P_{\mathrm{e}}$, are the cross-over error rates of the binary detection channel.

where $S_{\mathrm{vv}}(f)=\mathcal{F}\left\{R_{\mathrm{vv}}(z)\right\}$ is the power spectral density of $v(t)$ and $S_{\mathrm{vm}}(f)=$ $\mathcal{F}\left\{R_{\mathrm{vm}}(z)\right\}$ is the cross-spectral density of $m(t)$ and $s(t) . \mathcal{F}\{\}$ denotes the Fourier transform, which for a square integrable function $g$ is defined as

$$
\begin{aligned}
& G(f)=\mathcal{F}\{g(z)\}=\int_{-\infty}^{\infty} \mathrm{d} z g(z) \mathrm{e}^{-j 2 \pi f z}, \\
& g(t)=\mathcal{F}^{-1}\{G(f)\}=\int_{-\infty}^{\infty} \mathrm{d} f G(f) \mathrm{e}^{j 2 \pi f z} .
\end{aligned}
$$

We define a "reconstruction" noise for the signal estimation task as the difference between the stimulus and the optimal estimate $\hat{n}(t)=\hat{m}(t)-m(t)$. It is clear that $\mathcal{E}=\left\langle\hat{n}^{2}(t)\right\rangle$. Thus, performance in the estimation paradigm is quantified by $\mathcal{E}$; the lower the value of $\mathcal{E}$, the better the synapse is at transmitting the signal. The overall estimation paradigm (see Figure 1) can be abstracted by an effective continuous information channel shown in Figure 2A, where $m(t)$ is the input, $\hat{m}(t)$ the output, and $\hat{n}(t)$ the additive noise. The noise has zero mean, and its autocorrelation is given by $R_{\hat{n} \hat{n}}(z)=R_{\mathrm{mm}}(z)-\left(g \star R_{\mathrm{vm}}\right)(-z)$ (using equation 4.4$)$.

The power spectrum of the noise is given by,

$$
S_{\hat{\mathrm{n}} \hat{n}}(f)=\mathcal{F}\left\{R_{\hat{\mathrm{n}} \hat{n}}(z)\right\}=S_{\mathrm{mm}}(f)-\frac{\left|S_{\mathrm{vm}}(f)\right|^{2}}{S_{\mathrm{vv}}(f)} .
$$


Thus the MSE can be expressed as

$$
\mathcal{E}=\int_{\mathcal{S}} \mathrm{d} f S_{\hat{\mathrm{n}} \hat{n}}(f)=\sigma_{\mathrm{m}}^{2}-\int_{\mathcal{S}} \mathrm{d} f \frac{\left|S_{\mathrm{vm}}(f)\right|^{2}}{S_{\mathrm{vv}}(f)},
$$

where the set $\mathcal{S}=\left\{f \mid S_{\mathrm{vv}}(f) \neq 0\right\}$ is called the support of $S_{\mathrm{vv}}(f)$. As in Gabbiani and Koch (1998), we define a normalized measure called the coding fraction:

$$
\xi=1-\frac{\mathcal{E}}{\sigma_{\mathrm{m}}^{2}} .
$$

The coding fraction lies between 0 and $1,0 \leq \xi \leq 1$, where $\xi=0$ denotes chance performance and $\xi=1$ denotes perfect reconstruction. Using equation 4.7 , we can write

$$
\xi=\frac{1}{\sigma_{\mathrm{m}}^{2}} \int_{\mathcal{S}} \mathrm{d} f \frac{\left|S_{\mathrm{vm}}(f)\right|^{2}}{S_{\mathrm{vv}}(f)}
$$

Another measure of system performance is the mutual information rate $I(m ; v)$ between $m(t)$ and $v(t)$, defined as the amount of information transmitted by $v(t)$ about $m(t)$ in bits per second. By the data processing inequality (Cover \& Thomas, 1991), the mutual information between $m(t)$ and $v(t)$ is greater than the mutual information between $m(t)$ and $\hat{m}(t)$ :

$$
I(m ; v) \geq I(m ; \hat{m}) .
$$

A lower bound of $I(m ; \hat{m})$ and thus of $I(m ; v)$ has been derived in Gabbiani (1996) and Gabbiani and Koch (1996):

$$
I_{\mathrm{LB}}=\frac{1}{2} \int_{\mathcal{S}} \mathrm{d} f \log _{2}\left[\frac{S_{\mathrm{mm}}(f)}{S_{\mathrm{n} \hat{n}}(f)}\right] \quad\left(\text { in bit s }{ }^{-1}\right) .
$$

The lower bound is reached when the reconstruction noise $\hat{n}(t)$ is gaussian. Equation 4.9 differs from the expression for capacity of an additive gaussian channel model, commonly used in information theory (Cover \& Thomas, 1991), because, unlike in the case of the standard gaussian channel, the reconstruction noise $\hat{n}(t)$ (see Figure 2) is not independent of the input $m(t)$.

We further assume that the spike train $s(t)$ of the presynaptic neuron is a Poisson process with a mean firing rate, $\lambda(t)$, which is a function of the stimulus $m(t)$,

$$
\lambda(t)=\langle s(t)\rangle_{\mathrm{s} \mid \mathrm{m}}=f(k(t) \star m(t)),
$$

where $\langle\cdot\rangle_{s \mid \mathrm{m}}$ denotes an ensemble average over the spike train distribution for a fixed value of the stimulus $m(t)$. Since the stimulus itself is a stochastic 
(gaussian) process, the spike train $s(t)$ is called a doubly stochastic Poisson process. Although the Poisson assumption is not strictly valid for neural spike trains in general, it is fairly common in theoretical neuroscience because it allows for derivation of closed-form analytical expressions (Gabbiani, 1996; Gabbiani \& Koch, 1996).

$k(t)$ is a phenomenological filter that models the transformation of the stimulus modulations $m(t)$ into the neuron's firing rate, and $f(\cdot)$ is a static, memoryless nonlinearity that incorporates the nonlinear aspects of the neuron's input-output transformation, like half-wave rectification, saturation, and so on. The exact form of $k(t)$ is not important, although in order to derive closed-form expression later, we will assume that $k(t)$ has the form of a simple low-pass filter.

When the input-output transformation is linear, $f(x)=\bar{\lambda}+x^{2}$ the power spectrum of the spike train $s(t)$ can be expressed as

$$
S_{\mathrm{ss}}(f)=\bar{\lambda}+|K(f)|^{2} S_{\mathrm{mm}}(f),
$$

where $\bar{\lambda}$ is the mean firing rate and $K(f)$ is the Fourier transform of the filter $k(t)$. The variance of the firing rate $\sigma_{\lambda}^{2}$ is given by

$$
\sigma_{\lambda}^{2}=\int_{-\infty}^{\infty} d f|K(f)|^{2} S_{\mathrm{mm}}(f)
$$

We define the contrast of the firing rate as $c_{\lambda}=\sigma_{\lambda} / \bar{\lambda}$. The positivity of $\lambda(t)$ imposes a restriction on how large the contrast can be. For linear encoding, we will require that the mean firing rate be at least three times as large as the standard deviation of the firing-rate fluctuations, ensuring that the probability that $\lambda(t)$ is negative is less than 0.01 . This implies that $c_{\lambda} \leq 1 / 3$.

This presynaptic spike train is gated by a binary process corresponding to the stochastic vesicle release mechanism. Thus, the vesicle release is a Poisson process with rate $p \lambda(t)$ and $v(t)$ (see equation 4.1) is a filtered shot noise process. Its power spectrum is given by

$$
S_{\mathrm{vv}}(f)=|H(f)|^{2}\left[\left(\bar{q}^{2}+\sigma_{\mathrm{q}}^{2}\right) p \bar{\lambda}+\bar{q}^{2} p^{2}|K(f)|^{2} S_{\mathrm{mm}}(f)\right]+S_{\mathrm{nn}}(f) .
$$

The cross-spectral density $S_{\mathrm{vm}}(f)$ is given by

$$
S_{\mathrm{vm}}(f)=\bar{q} p S_{\mathrm{mm}}(f) H(f) K(f) .
$$

Using equations $4.8,4.13$, and 4.14 , we obtain

$$
\xi=\frac{1}{\sigma_{\mathrm{m}}^{2}} \int_{\mathcal{S}} \mathrm{d} f \frac{\left[S_{\mathrm{mm}}(f)\right]^{2}}{S_{\mathrm{mm}}(f)+S_{\text {neff }}(f)},
$$

\footnotetext{
${ }^{2}$ We assume that $\bar{\lambda}$ is high enough so that the probability of $\lambda(t)<0$ is negligible.
} 
where

$$
S_{\text {neff }}(f)=\frac{\bar{\lambda}\left(1+C V_{\mathrm{q}}^{2}\right)}{p|K(f)|^{2}}+\frac{S_{\mathrm{nn}}(f)}{p^{2} \bar{q}^{2}|H(f)|^{2}|K(f)|^{2}}
$$

Using equation 4.6 , the power spectrum of the reconstruction noise $\hat{n}(t)$ can be written as

$$
S_{\hat{\mathrm{n}} \hat{n}}(f)=\frac{S_{\mathrm{mm}}(f) S_{\mathrm{neff}}(f)}{S_{\mathrm{mm}}(f)+S_{\mathrm{neff}}(f)} .
$$

Synaptic transmission is ideal when the vesicle release is perfectly reliable, there is no variability in the EPSP amplitude, and membrane noise at the postsynaptic site is negligible $\left(p=1, C V_{\mathrm{q}}=0, \sigma_{\mathrm{n}}=0\right)$. The coding fraction corresponding to this ideal case is denoted by $\xi^{*}$, where

$$
\xi^{*}=\frac{1}{\sigma_{\mathrm{m}}^{2}} \int_{\mathcal{S}} \mathrm{d} f \frac{|K(f)|^{2} S_{\mathrm{mm}}^{2}(f)}{|K(f)|^{2} S_{\mathrm{mm}}(f)+\bar{\lambda}} .
$$

In general, even for perfect synaptic transmission, $\xi^{*}<1$ due to the stochastic Poisson nature of the spike train. For the parameter values we consider here (summarized in the caption of Figure 3 ), the second term in equation 4.16 can be neglected. The dominant first term in equation 4.16 indicates that signal estimation is limited by shot noise, that is, the inability to estimate the stimulus reliably is primarily due to the error in accurately estimating the firing rate of the neuron. It can be shown that for a fixed firing-rate contrast, as $\bar{\lambda} \rightarrow \infty, \xi \rightarrow 1$. In other words, perfect reconstruction takes place in the limit of infinite firing rates. This agrees well with intuition; since the stimulus modulations are linearly encoded in the firing rate $\lambda(t)$, an accurate estimate of $\lambda(t)$ can be deconvolved to recover the input. Thus, for the signal estimation task, synaptic unreliability and variability make it harder to estimate the firing rate from the postsynaptic voltage. As compared to the ideal case, the shot noise for a single synapse increases by a factor

$$
\kappa=\frac{1+C V_{\mathrm{q}}^{2}}{p} .
$$

To illustrate these results, we now apply them to a specific example modified from Gabbiani (1996). Let $m(t)$ be a white, band-limited signal over a bandwidth $B_{\mathrm{m}}$,

$$
\begin{aligned}
S_{\mathrm{mm}}(f) & =\frac{\sigma_{\mathrm{m}}^{2}}{2 B_{\mathrm{m}}} & & -B_{\mathrm{m}} \leq f \leq B_{\mathrm{m}} \\
& =0 & & \text { otherwise. }
\end{aligned}
$$


A
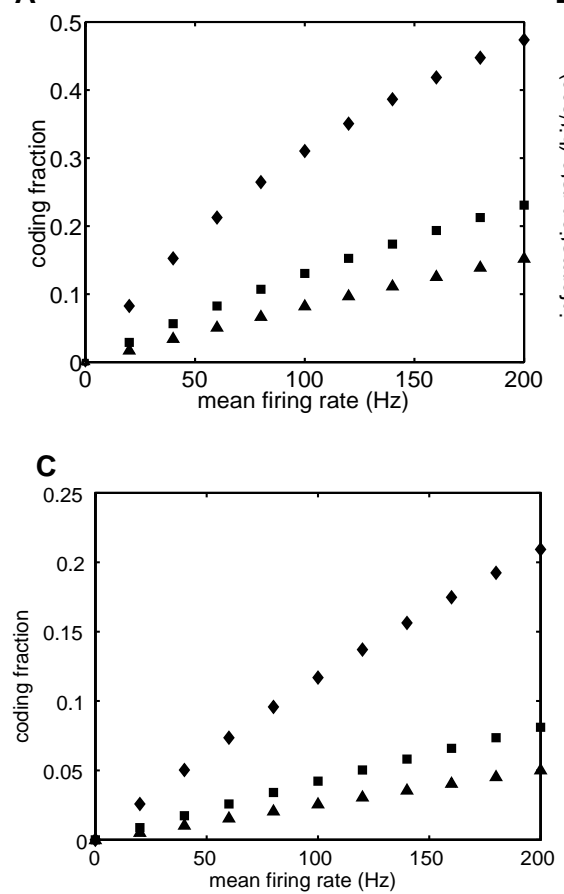

B

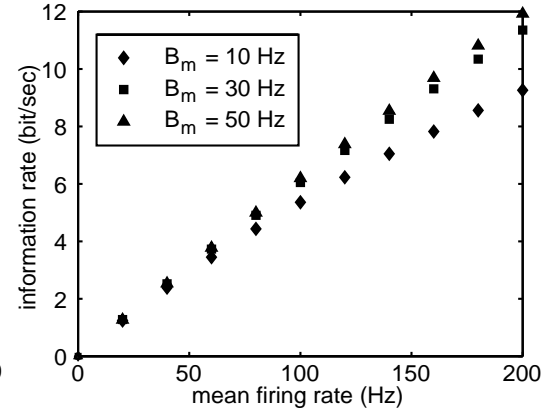

D

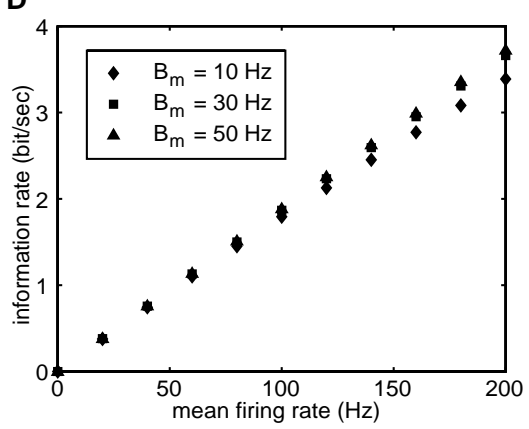

Figure 3: Comparison of performance in the signal estimation paradigm. (A) Coding fraction $\xi$ and (B) the lower bound on the information rate $I_{\mathrm{LB}}$ as a function of the mean firing rate $\bar{\lambda}$ for different stimulus bandwidths $B_{\mathrm{m}}$ for an ideal synapse $\left(p=1, C V_{\mathrm{q}}=0\right)$. (C) $\xi$ and (D) $I_{\mathrm{LB}}$ for a single unreliable and noisy synapse. We report the estimation performance for the optimal filter given by equations 4.21 and 4.23. Parameters are loosely based on those reported for cortical synapses: $\tau=20 \mathrm{msec}, c_{\lambda}=0.3, p=0.4, C V_{\mathrm{q}}=0.6, h_{\text {peak }}=1 \mathrm{mV}$, $t_{\text {peak }}=0.5 \mathrm{msec}, \sigma_{\mathrm{n}}=0.1 \mathrm{mV}, B_{\mathrm{n}}=100 \mathrm{~Hz}$.

If $k(t)$ is modeled as a simple low-pass filter, $k(t)=A \exp (-t / \tau)$,

$$
|K(f)|^{2}=\frac{A^{2} \tau^{2}}{1+(2 \pi f \tau)^{2}} .
$$

Using the above quantities, a closed-form expression for the coding fraction (denoted by $\xi_{\text {lp }}$ ) can be obtained (Gabbiani, 1996),

$$
\xi_{1 p}=\frac{\gamma}{\theta \sqrt{1+\gamma}} \tan ^{-1}\left(\frac{\theta}{\sqrt{1+\gamma}}\right)
$$


where

$$
\begin{aligned}
& \theta=2 \pi B_{\mathrm{m}} \tau \\
& \gamma=\frac{c_{\lambda}^{2} \bar{\lambda} \pi \tau}{\kappa \tan ^{-1} \theta} .
\end{aligned}
$$

$\theta$ is the ratio of the signal bandwidth to the filter's bandwidth, and $\gamma$ is the effective number of spikes available per unit signal bandwidth. As in Gabbiani (1996), one can also derive the optimal encoding filter that maximizes the coding fraction for a given stimulus power spectrum $S_{\mathrm{mm}}(f)$ under the constraint that the variance of the mean firing rate $\sigma_{\lambda}^{2}$ is fixed,

$$
|K(f)|^{2}=\frac{\sigma_{\lambda}^{2}}{\sigma_{\mathrm{m}}^{2}}, \quad-B_{\mathrm{m}} \leq f \leq B_{\mathrm{m}} .
$$

The coding fraction corresponding to the optimal filter above (denoted by $\left.\xi_{\text {opt }}\right)$ is given by

$$
\xi_{\mathrm{opt}}=\left(1+\frac{2 \kappa B_{\mathrm{m}}}{c_{\lambda}^{2} \bar{\lambda}}\right)^{-1} .
$$

Similarly, the lower bound for the low-pass filter $I_{\mathrm{LB}}$ can be obtained as

$$
\begin{aligned}
I_{\mathrm{LB}}=\frac{1}{2 \pi \tau \ln (2)}[ & \theta \ln \left(1+\frac{\gamma}{1+\theta^{2}}\right)+2 \sqrt{1+\gamma} \tan ^{-1}\left(\frac{\theta}{\sqrt{1+\gamma}}\right) \\
& \left.-2 \tan ^{-1} \theta\right]
\end{aligned}
$$

In equation 4.22 , In refers to the natural logarithm. The lower bound corresponding to the optimal encoding filter is given by

$$
\begin{aligned}
I_{\mathrm{LB}} & =B_{\mathrm{m}} \log _{2}\left(1+\frac{c_{\lambda}^{2} \bar{\lambda}}{2 \kappa B_{\mathrm{m}}}\right) \\
& =-B_{\mathrm{m}} \log _{2}\left(1-\xi_{\mathrm{opt}}\right) .
\end{aligned}
$$

Notice that when $\theta \ll 1, \xi_{\text {lp }} \rightarrow \xi_{\text {opt }}$, and the lower bound on the information rate $I_{\mathrm{LB}}$ for the low-pass filter and the optimal filter converge. Plots of $\xi$ and $I_{\mathrm{LB}}$ as functions of the mean firing rate $\bar{\lambda}$ and the input bandwidth $B_{\mathrm{m}}$ are shown in Figure 3. It can be observed that $\xi$ increases with $\bar{\lambda}$ and decreases with $B_{\mathrm{m}}$. This can be understood as follows: $\bar{\lambda} / B_{\mathrm{m}}$ denotes the average number of spikes available in the ideal case to estimate the mean firing rate of the spike train (thus the input) over a time period $\left(1 / B_{\mathrm{m}}\right)$ during which the input is relatively stationary. The fewer the number of spikes available, 
the poorer the estimate and lower the coding fraction. On the other hand, $I_{\mathrm{LB}}$ increases with $\bar{\lambda}$ and increases with $B_{\mathrm{m}}$ for low $B_{\mathrm{m}}$ but saturates at high $B_{\mathrm{m}}$. This is because the decrease in the quality of estimation ( $\xi$ decreases with $\left.B_{\mathrm{m}}\right)$ is compensated by an increase in the number of independent samples $\left(2 B_{\mathrm{m}}\right)$ transmitted per second. This can be observed by taking the limit $\bar{\lambda} \rightarrow 0$ (equivalently, $B_{\mathrm{m}} \rightarrow \infty$ ) in equation 4.23 ,

$$
\lim _{\bar{\lambda} \rightarrow 0} I_{\mathrm{LB}}=\frac{B_{\mathrm{m}}}{\ln 2} \frac{c_{\lambda}^{2} \bar{\lambda}}{2 \kappa B_{\mathrm{m}}}=\frac{1}{\ln 2} \frac{c_{\lambda}^{2} \bar{\lambda}}{2 \kappa},
$$

which is independent of $B_{\mathrm{m}}$. We refer to this condition as the low signal-tonoise regime. The information transmitted per spike is given by

$$
\lim _{\bar{\lambda} \rightarrow 0} \frac{I_{\mathrm{LB}}}{\bar{\lambda}}=\frac{c_{\lambda}^{2}}{2 \kappa \ln 2} .
$$

Notice that the maximum information per spike depends only on the contrast $c_{\lambda}$ of the firing-rate modulations and the factor $\kappa$. In the case of an ideal synapse for $c_{\lambda}=1 / 3$, the maximum information per spike is around 0.08 bits per spike. Notice that for a noisy synapse, the maximum information per spike decreases by a factor of $\kappa$. However, if we assume that the signal is encoded by a pair of halfwave rectifying neurons, each representing one-half (positive/negative) of the input $m(t)$, as in Gabbiani (1996) and Gabbiani and Koch (1996), the maximum amount of information that can be transmitted by an ideal synapse is approximately equal to 1.13 bits per spike. This corresponds to $c_{\lambda}=\sqrt{\pi / 2}(\approx 1.25)$. Thus, the low information rates we obtain here are a consequence of our assumption of linear encoding, which limits the magnitude of the firing-rate contrast. Nevertheless, the analysis can be readily extended to include other encoding schemes (integrate-andfire models, nonlinear Poisson encoding models with sigmoidal, half-wave rectification and other nonlinearities, and so on) and yield qualitatively similar results to those obtained here.

The analysis can be generalized to the case of multiple independent synaptic connections between two neurons. Let $N_{\text {syn }}$ denote the number of parallel synaptic connections between two neurons, which can occur in the form of $N_{\text {syn }}$ independent release sites driven by the same presynaptic process at the same postsynaptic location or as multiple synapses made at different locations. In either case, it is assumed that the vesicle release at a given synapse is statistically independent of the release at other synapses. However, this does not imply that the EPSP waveforms corresponding to the different synapses are independent. In fact, EPSPs are correlated since they are driven by the same presynaptic spike train $s(t)$. The postsynaptic membrane voltage can be written as

$$
V(t)=\sum_{l=1}^{N_{\mathrm{syn}}} \sum_{i} q_{i}^{l} W_{i}^{l} h^{l}\left(t-t_{i}\right)+n(t)
$$


where the superscript $l$ refers to the $l$ th synaptic connection. As before, we define $v(t)=V(t)-\langle V(t)\rangle$. If the synapses are distributed at different electrotonic locations on the postsynaptic neuron, the corresponding EPSP waveforms $h^{l}(t)$ are different. Similarly, if the release properties across the synaptic population are nonuniform (Rosenmund, Clements, \& Westbrook, 1993; Dobrunz \& Stevens, 1997), the random variables $\left(q_{i}^{l}, W_{i}^{l}\right)$ are governed by different distributions. However, for the sake of analytical tractability, we assume that the synapses are identical and are at the same electrotonic location. For the parameter values we consider here, the effect of the postsynaptic voltage noise $n(t)$ is negligible, and it can be shown that the shot noise increases from the ideal case by a factor,

$$
\kappa_{\mathrm{N}}=\frac{1}{N_{\mathrm{syn}}} \frac{1+C V_{\mathrm{q}}^{2}}{p}+\frac{N_{\mathrm{syn}}-1}{N_{\mathrm{syn}}} .
$$

The details of this derivation are provided in appendix B. In the limit of a large number of synapses $\left(N_{\text {syn }} \rightarrow \infty\right), \kappa_{N} \rightarrow 1$. Thus, the effect of synaptic unreliability and variability can be offset by redundancy in the number of synaptic connections between neurons. Plots of the coding fraction and the information rate for the signal estimation task as a function of the number of parallel synapses are shown in Figure 4 . Thus, although a single synapse has very low capacity, a small amount of redundancy causes a considerable increase in performance.

\section{Signal Detection}

We now consider the problem of detecting the presence of a presynaptic action potential from measurements of membrane voltage at the postsynaptic site. This signal detection paradigm frequently arises in different fields of science and engineering, like radar, digital communications, pattern recognition, and psychophysics. The objective in signal detection is to decide which member from a discrete set of signals was generated by a source, on the basis of measurements (possibly noisy) of its output. In the case we consider here, the set has two elements: the absence or presence of a presynaptic spike within a fixed temporal window. In our problem, the corresponding postsynaptic voltage waveform $V(t)$ measured over a period $0 \leq t \leq T$ corresponds to either the noise process $n(t)$ (denoted by hypothesis $H_{0}$ ) or a noisy version of the EPSP $h(t)$ gated by stochastic vesicle release $W$ (denoted by hypothesis $H_{1}$ ). Let $X$ and $Y$ be binary variables denoting occurrence of a presynaptic spike and the decision, respectively. Thus, $X=1$ if a spike occurred, else $X=0$. Similarly, $Y=1$ expresses the decision that a spike occurred. In psychophysics, the binary signal detection problem is known as a yes-no task (Green \& Swets, 1966). The detection paradigm has been used in neuroscience to explore the relationship between the outputs of individual cortical neurons and the performance of the animal in behavioral 
$\mathbf{A}$

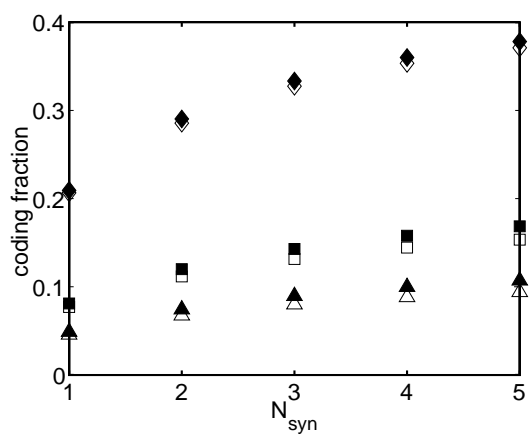

B

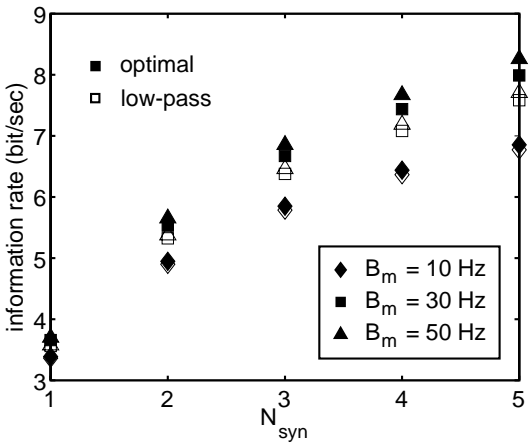

Figure 4: Effect of anatomical redundancy on estimation performance. (A) Coding fraction $\xi$ and (B) the information rate $I_{\mathrm{LB}}$ in signal estimation as a function of the number of parallel, identical synaptic connections $N_{\text {syn }}$ between a presynaptic and a postsynaptic neuron. The empty symbols correspond to performance in the presence of the low-pass filter, whereas the solid symbols correspond to the optimal encoding filter. There is little difference in estimation performance when $k(t)$ (see Figure 1) is chosen to be a low-pass filter and an optimal encoding filter matched to the stimulus. The performance saturates at high $N_{\text {syn }}$ as it approaches the performance of an ideal synapse $\left(p=1, C V_{\mathrm{q}}=0\right)$. The mean firing rate $\bar{\lambda}$ is $200 \mathrm{~Hz}$. Other parameter values used are summarized in the caption of Figure 3 .

tasks (Newsome et al., 1989; Britten et al., 1992; Shadlen \& Newsome, 1998). The binary signal detection problem can be formally expressed as

$$
\begin{aligned}
& \mathbf{H}_{\mathbf{0}}: V(t)=n(t), \quad \text { Noise } \\
& \mathbf{H}_{1}: V(t)=q W h(t)+n(t), \quad \text { Signal }+ \text { Noise }
\end{aligned}
$$

where $q$ is the random EPSP amplitude and $W$ is a binary variable representing the spike-conditioned vesicle release process. Thus, the goal is to design an optimal decision rule that minimizes the probability of error of detecting the presynaptic spike from the postsynaptic membrane voltage. Decision errors are of two kinds. A false alarm error $(\mathrm{F})$ occurs when the decision is in favor of the signal $(Y=1)$ when in fact noise was present $(X=0)$. Conversely, a miss error $(M)$ occurs when the decision is in favor of the noise $(Y=0)$ when the signal was present $(X=1)$. The probabilities of these errors are defined as

$$
P_{\mathrm{F}}=\operatorname{Prob}[Y=1 \mid X=0], \quad P_{\mathrm{M}}=\operatorname{Prob}[Y=0 \mid X=1] .
$$

The probability of detection error $P_{\mathrm{e}}$ is given by,

$$
P_{\mathrm{e}}=p_{0} P_{\mathrm{F}}+\left(1-p_{0}\right) P_{\mathrm{M}}
$$


where $p_{0}$ and $1-p_{0}$ are prior probabilities of occurrence of $H_{0}$ and $H_{1}$, respectively. We define a likelihood ratio $\Lambda_{X}(V)$ as

$$
\Lambda_{X}(V)=\frac{\operatorname{Prob}[V \mid X=1]}{\operatorname{Prob}[V \mid X=0]},
$$

where $\operatorname{Prob}[V \mid X=1]$ and $\operatorname{Prob}[V \mid X=0]$ denote the conditional probabilities of observing the voltage $V(t)$ conditioned on the presence and absence of a presynaptic spike, respectively. Using Bayes' rule, $\Lambda_{X}(V)$ can be expanded as

$$
\Lambda_{X}(V)=\frac{\operatorname{Prob}[X=1 \mid V]}{\operatorname{Prob}[X=0 \mid V]} \frac{p_{0}}{p_{1}},
$$

where $\operatorname{Prob}[X=1 \mid V]$ and $\operatorname{Prob}[X=0 \mid V]$ denote the posterior probabilities of the hypotheses conditioned on $V(t)$. The ratio

$$
\mathcal{L}(V)=\frac{\operatorname{Prob}[X=1 \mid V]}{\operatorname{Prob}[X=0 \mid V]}
$$

is referred to as the posterior likelihood, whereas, $\mathcal{L}_{0}=\left(1-p_{0}\right) / p_{0}$ is called the prior likelihood. The decision rule, which minimizes $P_{\mathrm{e}}$, is given by (Poor, 1994),

$$
\begin{array}{ll}
\mathcal{L}(V) \geq 1 & \Rightarrow Y=1 \\
\mathcal{L}(V)<1 & \Rightarrow Y=0,
\end{array}
$$

which can be written as

$$
\begin{aligned}
& \Lambda_{X}(V) \geq \mathcal{L}_{0}^{-1} \quad \Rightarrow Y=1 \\
& \Lambda_{X}(V)<\mathcal{L}_{0}^{-1} \quad \Rightarrow Y=0 .
\end{aligned}
$$

Using Bayes' rule, the spike conditional probabilities of the membrane voltage can be expressed in terms of release conditional probabilities as

$$
\begin{aligned}
& \operatorname{Prob}[V \mid X=1]=p \operatorname{Prob}[V \mid W=1]+(1-p) \operatorname{Prob}[V \mid W=0] \\
& \operatorname{Prob}[V \mid X=0]=\operatorname{Prob}[V \mid W=0] .
\end{aligned}
$$

As before, it is assumed that the spontaneous release probability is zero. The likelihood ratio $\Lambda_{\mathrm{X}}(V)$ can be expressed as

$$
\Lambda_{\mathrm{X}}(V)=p \Lambda_{\mathrm{W}}(V)+(1-p),
$$

where

$$
\Lambda_{W}(V)=\frac{\operatorname{Prob}[V \mid W=1]}{\operatorname{Prob}[V \mid W=0]} .
$$


In terms of $\Lambda_{\mathrm{W}}$, the optimal decision rule in equation 5.4 is given by

$$
\begin{array}{ll}
\Lambda_{\mathrm{W}}(V) \geq \frac{\left(\mathcal{L}_{0}^{-1}-1+p\right)}{p} & \Rightarrow Y=1 \\
\Lambda_{\mathrm{W}}(V)<\frac{\left(\mathcal{L}_{0}^{-1}-1+p\right)}{p} & \Rightarrow Y=0 .
\end{array}
$$

In the event of a vesicle release, the amplitude of the EPSP is a random variable drawn from a probability distribution $P(q)$ giving

$$
\Lambda_{\mathrm{W}}(V)=\int \mathrm{d} q P(q) \frac{\operatorname{Prob}[V \mid q ; W=1]}{\operatorname{Prob}[V \mid W=0]},
$$

where $\operatorname{Prob}[V \mid q ; W=1]$ is the probability distribution of the postsynaptic voltage conditioned on the quantal amplitude of a spike-triggered vesicle release. If $n(t)$ is a white gaussian noise process with a sufficiently large bandwidth $B_{\mathrm{n}}$, which satisfies $B_{\mathrm{n}} t_{\text {peak }} \gg 1$, equation 5.6 can be simplified as (Helstrom, 1968)

$$
\Lambda_{\mathrm{W}}(V)=\int \mathrm{d} q P(q) \exp \left(2 q r_{\mathrm{T}} \sqrt{S N R_{\mathrm{T}}}-q^{2} S N R_{\mathrm{T}}\right),
$$

where

$$
S N R_{\mathrm{T}}=\frac{1}{S_{\mathrm{nn}}(f)} \int_{0}^{T} \mathrm{~d} t h^{2}(t), \quad r_{\mathrm{T}}=\frac{\int_{0}^{T} \mathrm{~d} t h(t) V(t)}{\sqrt{S_{\mathrm{nn}}(f) \int_{0}^{T} \mathrm{~d} t h^{2}(t)}} .
$$

The temporal support of $h(t)$ is very small (on the order of a few milliseconds), and so $S N R_{\mathrm{T}}$ quickly saturates with $T$. Thus, when the interval length $T$ is greater than a few milliseconds (which we shall assume here), $S N R_{\mathrm{T}} \approx S N R$.

When $q$ is a one-sided random variable (here $q$ is positive), it can be shown that $\Lambda_{W}$ is a monotonic function of $r_{\mathrm{T}}$. Thus, the decision rule in equation 5.5 reduces to

$$
\begin{array}{ll}
r_{\mathrm{T}} \geq \Theta & \Rightarrow Y=1 \\
r_{\mathrm{T}}<\Theta & \Rightarrow Y=0 .
\end{array}
$$

where the threshold $\Theta$ depends on $\mathcal{L}_{0}, p, P(q)$, and so on. Thus, the optimal decision rule involves comparing the correlation, $r_{\mathrm{T}}$, between $V(t)$ and $h(t)$ (over the time interval T), to a threshold $\Theta$ and deciding $Y=1$ if $r_{\mathrm{T}}$ exceeds the threshold and $Y=0$ otherwise. The error probabilities $\left(P_{\mathrm{F}}\right.$ and $\left.P_{\mathrm{M}}\right)$ can be written as

$$
\begin{aligned}
P_{\mathrm{F}} & =\operatorname{Prob}\left[r_{\mathrm{T}} \geq \Theta \mid X=0\right]=\operatorname{Prob}\left[r_{\mathrm{T}} \geq \Theta \mid W=0\right] \\
P_{\mathrm{M}} & =\operatorname{Prob}\left[r_{\mathrm{T}}<\Theta \mid X=1\right] \\
& =p \operatorname{Prob}\left[r_{\mathrm{T}}<\Theta \mid W=1\right]+(1-p) \operatorname{Prob}\left[r_{\mathrm{T}}<\Theta \mid W=0\right] .
\end{aligned}
$$


Let $P_{\mathrm{F}}^{0}\left(\operatorname{Prob}\left[r_{\mathrm{T}} \geq \Theta \mid W=0\right]\right)$ and $P_{\mathrm{M}}^{0}\left(\operatorname{Prob}\left[r_{\mathrm{T}}<\Theta \mid W=1\right]\right)$ denote the corresponding errors when the vesicle release is deterministic and no spontaneous release occurs. $P_{\mathrm{F}}$ and $P_{\mathrm{M}}$ can be expressed in terms of $P_{\mathrm{F}}^{0}$ and $P_{\mathrm{M}}^{0}$ as

$$
P_{\mathrm{F}}=P_{\mathrm{F}}^{0}, \quad P_{\mathrm{M}}=P_{\mathrm{M}}^{0}+(1-p)\left(1-P_{\mathrm{M}}^{0}-P_{\mathrm{F}}^{0}\right) .
$$

When the postsynaptic noise $n(t)$ is negligible $(S N R \rightarrow \infty)$, both the conditional errors $P_{\mathrm{F}}^{0}$ and $P_{\mathrm{M}}^{0} \rightarrow 0$. However, in the limit of no postsynaptic voltage noise, $P_{\mathrm{M}} \rightarrow(1-p)$ due to the unreliability of vesicle release. Let $P_{\mathrm{e}}^{*}=\left(1-p_{0}\right)(1-p)$ denote the minimum possible detection error when $S N R \rightarrow \infty$. The probability of error $P_{\mathrm{e}}$ can be written as

$$
P_{\mathrm{e}}=P_{\mathrm{e}}^{*}+P_{\mathrm{F}}^{0}\left[p_{0}-\left(1-p_{0}\right)(1-p)\right]+P_{\mathrm{M}}^{0}\left(1-p_{0}\right) p .
$$

Since $r_{\mathrm{T}}$ is a conditional gaussian random whose distribution depends on the value of the release variable $W$, the conditional means and variances of $r_{\mathrm{T}}$ can be derived as

$$
\begin{aligned}
& \left\langle r_{\mathrm{T}}\right\rangle_{0}=0 \quad\left\langle r_{\mathrm{T}}\right\rangle_{1, \mathrm{q}}=q \sqrt{S N R} \\
& \left\langle r_{\mathrm{T}}^{2}\right\rangle_{0}=\left\langle\left(r_{\mathrm{T}}-q \sqrt{S N R}\right)^{2}\right\rangle_{1, \mathrm{q}}=1,
\end{aligned}
$$

where $\langle\cdot\rangle_{0}$ denotes an ensemble average conditioned on $W=0$ and $\langle\cdot\rangle_{1, q}$ denotes an ensemble average conditioned on $W=1$ and amplitude $q$. Thus, $P_{\mathrm{F}}^{0}$ and $P_{\mathrm{M}}^{0}$ can be parametrically expressed in terms of the threshold $\Theta$,

$$
\begin{aligned}
P_{\mathrm{F}}^{0} & =\frac{1}{2}[1-\operatorname{Erf}(\Theta)] \\
P_{\mathrm{M}}^{0} & =\frac{1}{2}\left[1+\int_{0}^{\infty} \mathrm{d} q P(q) \operatorname{Erf}(\Theta-q \sqrt{S N R})\right]
\end{aligned}
$$

where $\operatorname{Erf}(x)$ is the error function defined as $\operatorname{Erf}(x)=2 / \sqrt{\pi} \int_{0}^{x} \mathrm{~d} t \exp \left(-t^{2}\right)$.

In general, it is not possible to derive closed-form expressions for $P_{\mathrm{M}}^{0}$. Both $P_{\mathrm{F}}^{0}$ and $P_{\mathrm{M}}^{0}$ depend on the choice of the threshold $\Theta$. If $\Theta$ is large, $P_{\mathrm{F}}^{0}$ is low but $P_{\mathrm{M}}^{0}$ is high, whereas if $\Theta$ is small, $P_{\mathrm{M}}^{0}$ is low but $P_{\mathrm{F}}^{0}$ is high. Thus, as $\Theta$ is varied, the probability of error $P_{\mathrm{e}}$ goes through a minimum. By plotting $P_{\mathrm{e}}$ as a function of the threshold, we can graphically obtain the optimal value of $\Theta$, which minimizes $P_{\mathrm{e}}$ (see Figure 5). We can model the detection task as a binary information channel with the binary variable $X$ as its input and the decision $Y$ as the output. The error probabilities $P_{\mathrm{F}}$ and $P_{\mathrm{M}}$ denote the cross-over probabilities of the binary channel shown in Figure 2B. Performance in the detection task can be quantified by either $P_{\mathrm{e}}$ or the mutual information between the binary random variables, $X$ and $Y$, 
A

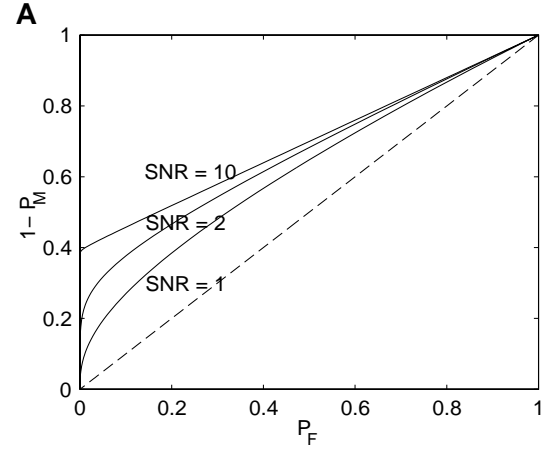

C

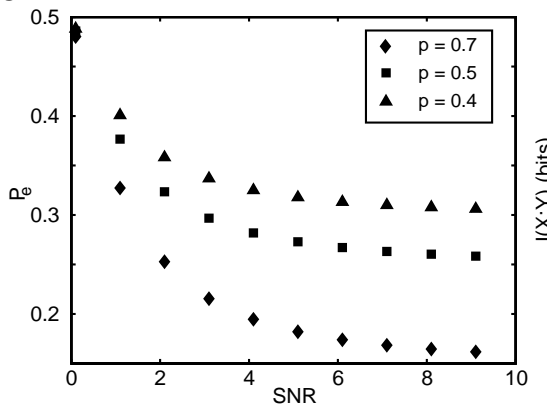

B

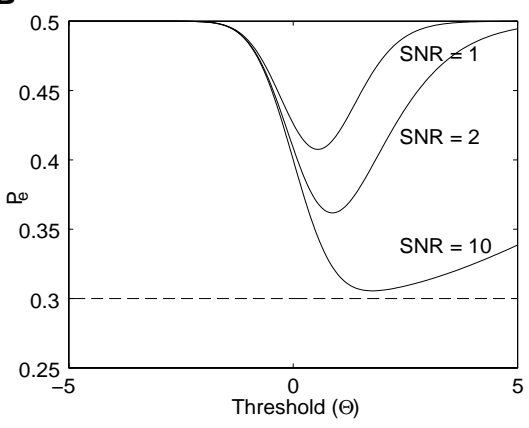

D

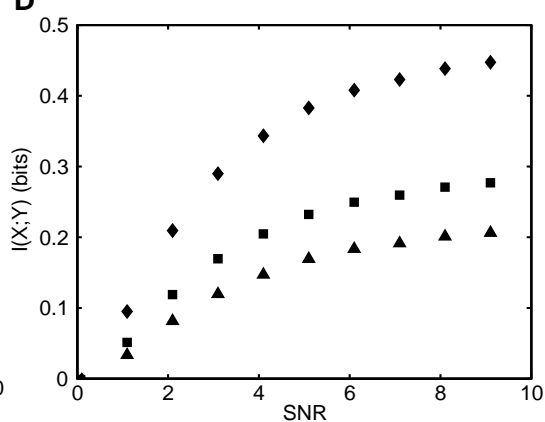

Figure 5: Comparison of performance in the signal detection paradigm. (A) Plot of detection error $\left(1-P_{\mathrm{M}}\right)$ versus false alarm error $P_{\mathrm{F}}$ for a single synapse as the threshold $\Theta$, used to detect a presynaptic action potential, is varied from $-\infty$ to $\infty$. The curves correspond to different values of the signal-to-noise ratio SNR, a measure of the size of the EPSP peak relative to the postsynaptic noise magnitude. The dashed diagonal line corresponds to chance performance (SNR $=0$ ). Parameters: $p=0.4$. See below for a summary of the other parameters. (B) Probability of error $P_{\mathrm{e}}=0.5 P_{\mathrm{F}}+0.5 P_{\mathrm{M}}$ (prior probability of a spike $p_{0}=$ $0.5)$ plotted against $\Theta$ to determine the optimal value of the threshold, which minimizes $P_{\mathrm{e}}$. The dashed horizontal line corresponds to the minimum possible detection error for the synaptic parameters in the limit SNR $\rightarrow \infty$. (C) Plot of the optimal $P_{\mathrm{e}}$ versus SNR for different values of the synaptic release probability $p$. The optimal $P_{\mathrm{e}}$ for a given SNR is given by the minimum of the corresponding curve in $B$. The curves saturate at high SNR to their minimum values $P_{\mathrm{e}}^{*}=$ $\left(1-p_{0}\right)(1-p)$. (D) Plot of the mutual information $I(X ; Y)$ in the detection task as a function of SNR. Parameter values: $\bar{q}=1, C V_{\mathrm{q}}=0.6, h_{\text {peak }}=2 \mathrm{mV}, t_{\text {peak }}=$ $1 \mathrm{msec}, B_{\mathrm{n}}=100 \mathrm{~Hz}$. 
A

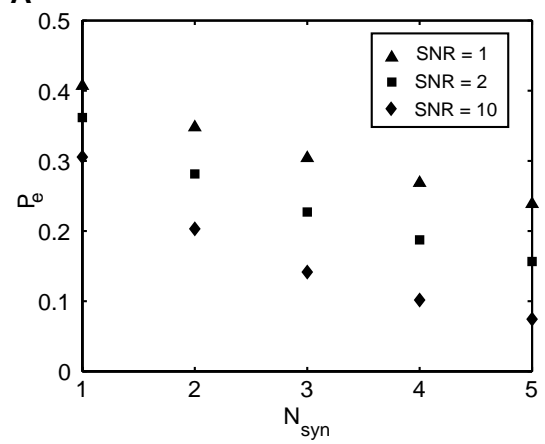

B

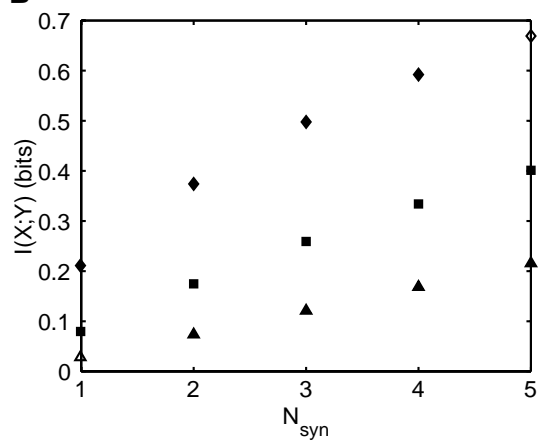

Figure 6: Effect of anatomical redundancy on detection performance. (A) Probability of error $P_{\mathrm{e}}$ and (B) the mutual information between $X$ and $Y I(X ; Y)$ as a function of the number of synapses $N_{\text {syn }}$ for the signal detection task for different values of SNR. Synaptic parameters: $p=0.4$; other parameters are summarized in the Figure 5 caption.

denoted by $I(X ; Y)$. The synapse is ideal when $P_{\mathrm{e}}=0$ and $I(X ; Y)=1$ bit, and at chance, when $P_{\mathrm{e}}=0.5$ and $I(X ; Y)=0$ bits. $I(X ; Y)$ can be computed using the formula for the mutual information for a binary channel (Cover \& Thomas, 1991),

$$
I(X ; Y)=\mathcal{H}\left(P_{Y}\right)-p_{0} \mathcal{H}\left(P_{\mathrm{F}}\right)-\left(1-p_{0}\right) \mathcal{H}\left(P_{\mathrm{M}}\right)
$$

where $P_{\mathrm{Y}}=p_{0}\left(1-P_{\mathrm{F}}\right)+\left(1-p_{0}\right) P_{\mathrm{M}}$ and $\mathcal{H}(x)=-x \log _{2}(x)-(1-x) \log _{2}(1-x)$ is the binary entropy function (Cover \& Thomas, 1991).

The analysis can be generalized to the case of $N_{\text {syn }}$ independent parallel synapses (see appendix C). Plots of $P_{\mathrm{e}}$ and $I(X ; Y)$ versus $N_{\text {syn }}$ for different values of $S N R$ for the case of identical synapses are shown in Figures 6A and $6 \mathrm{~B}$, respectively. Once again, we observe the poor performance of a single synapse and the substantial improvement due to anatomical redundancy. The linear increase of $I$ with $N_{\text {syn }}$ is similar to the result obtained for signal estimation.

\section{Discussion}

We have designed a simple mathematical model of a cortical synapse that incorporates the known unreliable and variable nature of central synapses. By assessing the performance of this model for signal estimation and signal detection tasks, we have shown that single synapses (meant to mimic a central synapse in cortex) are relatively ineffective at transmitting information between neurons. However, only a small amount of redundancy in the number of synaptic connections is sufficient to render synaptic trans- 
mission more robust and reliable. These results are valid for both the signal estimation and signal detection tasks.

In order to obtain analytical expressions for measures of synaptic efficacy, we made some simplifying assumptions. In our model, the release probability of the synapse was assumed to be constant. This is a gross oversimplification since it has been shown that the release probability can be strongly modulated by the pattern of presynaptic spike trains and can in fact vary over a large range (Dobrunz \& Stevens, 1999). In fact, cortical synapses exhibit history-dependent plasticity over a variety of timescales (Markram \& Tsodyks, 1996; Abbott et al., 1997; Dobrunz \& Stevens, 1997; Dobrunz, Huang, \& Stevens, 1997). Additionally, there is a significant nonuniformity in the release probabilities across synapses onto the same neuron (Hessler et al., 1993; Rosenmund et al., 1993; Dobrunz \& Stevens, 1999), though we assume synaptic parameters to be the same at all synapses. These assumptions allowed us to derive closed-form expressions of the amount of information that can be transmitted across noisy synapses. Moreover, our conclusions depend on the signal and synaptic parameters and thus need not hold for synapses whose properties depart strongly from our choice of parameter values. Although the parameters here have been chosen mainly to demonstrate the applicability of our analysis, the generality of the closed-form expressions also allows assessment of synaptic efficacy when the parameters can be obtained from empirical data.

Our results agree qualitatively with a recent study that investigated the influence of synaptic unreliability on information transfer (Zador, 1998), though the information rates we obtain are much smaller in magnitude. This is a consequence of our assumption that the encoding relationship between the mean firing rate of the presynaptic neuron and the input is linear. This assumption constrains the contrast of the firing rate $c_{\lambda}$ (less than one-third) and limits the amount of information that can be transmitted by the spike train over the ideal synapse (see Figures 3A and 3B). Moreover, our spike encoding model is a doubly stochastic Poisson process with a stimulus-dependent mean firing rate (i.e., a random stimulus with zero mean is encoded into a Poisson distributed spike train that is sent through a binary channel) as compared to the leaky integrate-and-fire model used in Zador (1998) to transform the continuous input to a spike train. It can be shown that for the same mean firing rate, the leaky integrate-and-fire model can transmit much more information than a Poisson process, since the latter is more random than the former. If we normalize the synaptic information rates with respect to the corresponding rates for the ideal synapse (using the spike train directly), we obtain the same behavior for a host of different encoding models. We chose the Poisson model mainly for reasons of analytical tractability.

This study represents our continuing efforts to resolve information transmission between neurons in terms of the contributions of the different biophysical components that constitute the neuronal link rather than accurately 
estimating neuronal capacity. Our approach is different from some of the other techniques used to decode the nature of the neural code. Bialek and colleagues (Rieke et al., 1997) pioneered the reconstruction technique to quantify the coding efficiency of spiking neurons and applied it to understand the nature of neural codes in a variety of biological systems. Model-independent methods that directly estimate the information capacity of spiking neurons have also been developed and applied recently (DeWeese \& Bialek, 1995; Stevens \& Zador, 1996; Strong, Koberle, van Steveninck, \& Bialek, 1998). Our research program is driven by the hypothesis that noise fundamentally limits the precision, speed, and accuracy of computation in the nervous system (Koch, 1999). Thus, assessing the role of each biophysical stage in information transfer requires a characterization of the corresponding sources of variability that can cause loss of signal fidelity. In this context, we studied the effect of synaptic unreliability on the amount of information transmitted across synaptic connections. In earlier studies, we investigated how neuronal membrane noise sources influence and ultimately limit the ability of noisy dendrites to transmit information from the synaptic location to the soma (Manwani \& Koch, 1999a, 1999b). The validity of our theoretical results needs to be assessed by comparing experimental data obtained from a well-characterized neurobiological system. We are in the process of analyzing these noise sources using detailed biophysical models and studying the effect of the measured noise on the temporal precision of spike firing in anatomically and physiologically characterized neuronal models. This will allow us to estimate the information capacity of these neurons and explore the possible functional role of noise.

\section{Appendix A: List of Symbols}

\begin{tabular}{ll}
\hline Symbol & Description \\
\hline$\sigma_{\lambda}$ & Standard deviation of the presynaptic neuron's firing rate \\
$\sigma_{\mathrm{m}}$ & Standard deviation of input signal in signal estimation \\
$\sigma_{\mathrm{n}}$ & Standard deviation of postsynaptic membrane noise \\
$\sigma_{\mathrm{n}}$ & Standard deviation of quantal amplitude \\
$\tau$ & Time constant of encoding filter in signal estimation \\
$\xi$ & Normalized coding fraction in signal estimation \\
$B_{\mathrm{m}}$ & Bandwidth of input signal in signal estimation \\
$B_{\mathrm{n}}$ & Bandwidth of postsynaptic membrane noise \\
$c_{\lambda}$ & Contrast of the presynaptic neuron's firing rate \\
$C V_{\mathrm{q}}$ & Coefficient of variation of quantal amplitude \\
$h(t)$ & Shape of the EPSP waveform \\
$h_{\text {peak }}$ & Peak of the EPSP waveform \\
$k(t)$ & Linear encoding filter in signal estimation \\
$K(f)$ & Fourier transform of $k(t)$ \\
$I(X ; Y)$ & Mutual information for signal detection
\end{tabular}




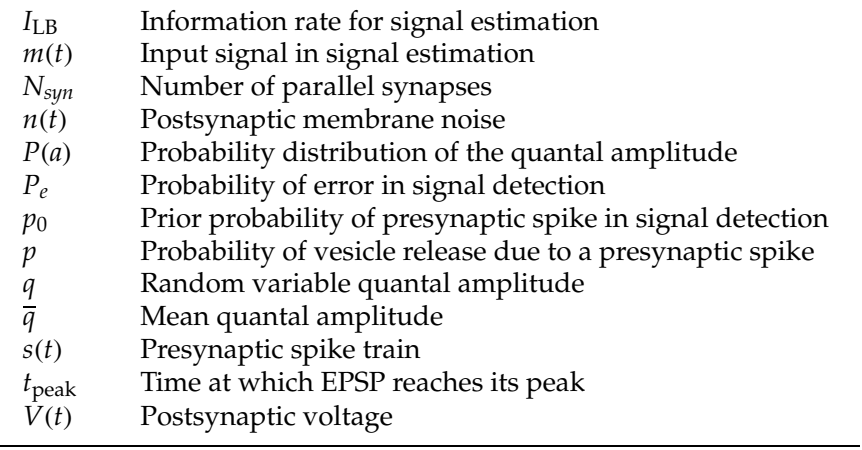

\section{Appendix B: Estimation with Multiple Synapses}

In the case of multiple synapses, the postsynaptic membrane voltage is given by equation 4.25 . We assume that the EPSP waveforms corresponding to the different synapses are identical, $h^{l}(t)=h(t), \forall l$ :

$$
V(t)=\sum_{l=1}^{N_{\text {syn }}} \sum_{i} q_{i}^{l} W_{i}^{l} h\left(t-t_{i}\right)+n(t) .
$$

As before, the postsynaptic noise $n(t)$ is assumed to be independent of the input $m(t)$ and the presynaptic release processes. The cross-spectrum between the zero-mean membrane voltage $v(t)$ and $m(t)$ for $N_{\text {syn }}$ synapses is a scaled version of the cross-spectrum for a single synapse, equation 4.14:

$$
S_{\mathrm{vm}}(f)=N_{\text {syn }} p \bar{q} K(f) H(f) S_{\mathrm{mm}}(f) .
$$

The autocorrelation function for the membrane voltage can be computed as follows:

$$
\begin{aligned}
R_{\mathrm{vv}}(\tau) & =\langle V(t) V(t+\tau)\rangle) \\
& =\left\langle\sum_{l=1}^{N_{\mathrm{syn}}} \sum_{m=1}^{N_{\mathrm{syn}}} \sum_{i} \sum_{j} q_{i}^{l} q_{j}^{m} W_{i}^{l} W_{j}^{m} h\left(t-t_{i}\right) h\left(t+\tau-t_{j}\right)\right\rangle .
\end{aligned}
$$

The above expression can be simplified as a sum of the following four terms:

$$
\begin{aligned}
R_{\mathrm{vv}}(\tau)= & \left\langle\sum_{l=1}^{N_{\mathrm{syn}}} \sum_{i}\left(q_{i}^{l}\right)^{2}\left(W_{i}^{l}\right)^{2} h\left(t-t_{i}\right) h\left(t+\tau-t_{i}\right)\right\rangle \\
& +\left\langle\sum_{l=1}^{N_{\mathrm{syn}}} \sum_{i} \sum_{j, j \neq i} q_{i}^{l} q_{j}^{l} W_{i}^{l} W_{j}^{l} h\left(t-t_{i}\right) h\left(t+\tau-t_{j}\right)\right\rangle
\end{aligned}
$$




$$
\begin{aligned}
& +\left\langle\sum_{l=1}^{N_{\mathrm{syn}}} \sum_{m=1, m \neq l}^{N_{\mathrm{syn}}} \sum_{i} q_{i}^{l} q_{i}^{m} W_{i}^{l} W_{i}^{m} h\left(t-t_{i}\right) h\left(t+\tau-t_{i}\right)\right\rangle \\
& +\left\langle\sum_{l=1}^{N_{\mathrm{syn}}} \sum_{m=1, m \neq l}^{N_{\mathrm{syn}}} \sum_{i} \sum_{j, j \neq i} q_{i}^{l} q_{j}^{m} W_{i}^{l} W_{j}^{m} h\left(t-t_{i}\right) h\left(t+\tau-t_{j}\right)\right\rangle .
\end{aligned}
$$

We assume that $q_{i}^{l}$ and $W_{i}^{l}$ are independent and identical random variables corresponding. Thus,

$$
\left\langle q_{i}^{l} q_{j}^{m}\right\rangle=\bar{q}^{2}+\sigma_{\mathrm{q}}^{2} \delta_{l m} \delta_{i j} \quad\left\langle W_{i}^{l} W_{j}^{m}\right\rangle=p^{2}+p(1-p) \delta_{l m} \delta_{i j}
$$

where $\delta_{i j}$ denotes the Kronecker delta function. Using these expressions, $R_{\mathrm{vv}}(\tau)$ can be simplified as

$$
\begin{aligned}
R_{\mathrm{vv}}(\tau)= & N_{\text {syn }} p\left(\bar{q}^{2}+\sigma_{\mathrm{q}}^{2}\right)\left\langle\sum_{i} h\left(t-t_{i}\right) h\left(t+\tau-t_{i}\right)\right\rangle \\
& +N_{\mathrm{syn}} p^{2} \bar{q}^{2}\left\langle\sum_{i} \sum_{j, j \neq i} h\left(t-t_{i}\right) h\left(t+\tau-t_{j}\right)\right\rangle \\
& +N_{\mathrm{syn}}\left(N_{\mathrm{syn}}-1\right) p^{2} \bar{q}^{2}\left\langle\sum_{i} h\left(t-t_{i}\right) h\left(t+\tau-t_{i}\right)\right\rangle \\
& +N_{\mathrm{syn}}\left(N_{\mathrm{syn}}-1\right) p^{2} \bar{q}^{2}\left\langle\sum_{i} \sum_{j, j \neq i} h\left(t-t_{i}\right) h\left(t+\tau-t_{j}\right)\right\rangle .
\end{aligned}
$$

Using the following expressions,

$$
\begin{gathered}
\left\langle\sum_{i} h\left(t-t_{i}\right) h\left(t+\tau-t_{i}\right)\right\rangle=\bar{\lambda} h(\tau) \star h(-\tau) \\
\left\langle\sum_{i} \sum_{j, j \neq i} h\left(t-t_{i}\right) h\left(t+\tau-t_{j}\right)\right\rangle=h(\tau) \star h(-\tau) \star k(\tau) \star k(-\tau) \\
\star R_{m m}(\tau),
\end{gathered}
$$

the power spectrum of $v(t)$ can be obtained by taking the Fourier transform of $R_{\mathrm{vV}}(\tau)$ :

$$
\begin{aligned}
S_{\mathrm{vv}}(f)= & N_{\text {syn }}^{2} \bar{q}^{2} p^{2}|K(f)|^{2}|H(f)|^{2} S_{\mathrm{mm}}(f) \\
& +N_{\mathrm{syn}} p \bar{\lambda}|H(f)|^{2}\left[\left(\bar{q}^{2}+\sigma_{\mathrm{q}}^{2}\right)+\left(N_{\mathrm{syn}}-1\right) \bar{q}^{2} p\right]+S_{\mathrm{nn}}(f) .
\end{aligned}
$$


Substituting for $S_{\mathrm{vm}}(f)$ and $S_{\mathrm{vv}}(f)$ in equation 4.8 , the coding fraction $\xi$ can be written as

$$
\xi=\frac{1}{\sigma_{\mathrm{m}}^{2}} \int_{\mathcal{S}} \mathrm{d} f \frac{\left[S_{\mathrm{mm}}(f)\right]^{2}}{S_{\mathrm{mm}}(f)+S_{\text {neff }}(f)},
$$

where

$$
S_{\text {neff }}(f)=\frac{\bar{\lambda}}{|K(f)|^{2}}\left[\frac{\left(1+C V_{\mathrm{q}}^{2}\right)}{p N_{\text {syn }}}+\frac{N_{\text {syn }}-1}{N_{\text {syn }}}\right]+\frac{S_{\text {nn }}(f)}{N_{\text {syn }}^{2} p^{2} \bar{q}^{2}|H(f)|^{2}|K(f)|^{2}} .
$$

Assuming that the second term in the above expression is negligible, in comparison to the ideal case, the shot noise is multiplied by a factor

$$
\kappa_{\mathrm{N}}=\frac{1}{N_{\mathrm{syn}}} \frac{1+C V_{\mathrm{q}}^{2}}{p}+\frac{N_{\mathrm{syn}}-1}{N_{\mathrm{syn}}} .
$$

Note that $\kappa_{\mathrm{N}} \rightarrow 1$ in the limit of a large number of synapses, $N_{\mathrm{syn}} \rightarrow \infty$.

\section{Appendix C: Detection with Multiple Synapses}

It can be shown that the optimal decision rule for multiple synapses also involves comparing the correlation between the membrane voltage $V(t)=$ $\sum_{l=1}^{N_{\text {syn }}} q^{l} W^{l} h(t)+n(t)$ and the EPSP shape $h(t)$ to a threshold $\Theta$. The random variable $r$ can be written as

$$
r=q_{\mathrm{N}} \sqrt{S N R}+\frac{\int \mathrm{d} t h(t) n(t)}{\sqrt{S_{\mathrm{nn}}(f) \int \mathrm{d} t h^{2}(t)}},
$$

where $q_{\mathrm{N}}=\sum_{l=1}^{N_{\mathrm{syn}}} q^{l} W^{l}$. Since no spontaneous vesicle release occurs, $q_{\mathrm{N}} \equiv 0$ in the absence of a presynaptic spike $(X=0)$. In this case, $r$ is a zero-mean, unit variance gaussian random variable with probability density

$$
\operatorname{Prob}[r \mid X=0]=\frac{1}{\sqrt{2 \pi}} \exp \left(\frac{-r^{2}}{2}\right)
$$

However, when a presynaptic action potential occurs $(X=1), q_{\mathrm{N}}$ is a random variable, which depends on the number of synapses at which vesicle release occurs and the postsynaptic magnitude subsequent to a release. The spikeconditioned density of $r$ is given by

$$
\operatorname{Prob}[r \mid X=1]=\frac{1}{\sqrt{2 \pi}} \int_{0}^{\infty} \mathrm{d} q_{\mathrm{N}} P\left(q_{\mathrm{N}}\right) \exp \left[\frac{-\left(r-q_{\mathrm{N}} \sqrt{S N R}\right)^{2}}{2}\right],
$$


where $P_{N}\left(q_{N}\right)$ is the probability density of $q_{N}$. Since $q_{N}$ is a sum of independent identical random variables, $P\left(q_{\mathrm{N}}\right)$ can be obtained by convolving the probability density of the random variable $q_{1}=q W$ with itself $N_{\text {syn }}$ times. Thus,

$$
P\left(q_{\mathrm{N}}\right)=\underbrace{P_{1}\left(q_{1}\right) \star P_{1}\left(q_{1}\right) \cdots \star P_{1}\left(q_{1}\right)}_{N_{\text {syn }} \text { times }},
$$

where

$$
P_{1}\left(q_{1}\right)=(1-p) \delta\left(q_{1}\right)+p P(q) \text {. }
$$

In the equation above, $(1-p) \delta\left(q_{1}\right)$ denotes the probability mass at $q_{1}=0$ corresponding to release failure, and $P(q)$ is the postsynaptic EPSP amplitude distribution when a single release occurs. Thus, the error probabilities $\left(P_{\mathrm{F}}\right.$ and $\left.P_{\mathrm{M}}\right)$ can be written as

$$
\begin{aligned}
P_{\mathrm{F}} & =\operatorname{Prob}\left[r_{\mathrm{T}} \geq \Theta \mid X=0\right]=\frac{1}{\sqrt{2 \pi}} \int_{\Theta}^{\infty} \mathrm{d} r \exp \left(\frac{-r^{2}}{2}\right) \\
P_{\mathrm{M}} & =\operatorname{Prob}\left[r_{\mathrm{T}}<\Theta \mid X=1\right] \\
& =\frac{1}{\sqrt{2 \pi}} \int_{-\infty}^{\Theta} \mathrm{d} r \int_{0}^{\infty} \mathrm{d} q_{\mathrm{N}} P_{N}\left(q_{\mathrm{N}}\right) \exp \left[\frac{-\left(r-q_{\mathrm{N}} \sqrt{S N R}\right)^{2}}{2}\right] .
\end{aligned}
$$

\section{Acknowledgments}

This research was supported by NSF, NIMH, and the Sloan Center for Theoretical Neuroscience. We thank Fabrizio Gabbiani, Tony Zador, and Peter Steinmetz for illuminating discussions.

\section{References}

Abbott, L. F., Varela, J. A., Sen, K., \& Nelson, S. B. (1997). Synaptic depression and cortical gain-control. Science, 275, 220-224.

Abeles, M. (1990). Corticonics: Neural circuits of the cerebral cortex. New York: Cambridge University Press.

Ackley, D. H., Hinton, G. E., \& Sejnowski, T. J. (1985). A learning algorithm for Boltzman machines. Cog. Sci., 9, 147-169.

Adrian, E. D. (1932). The mechanism of nervous action: Electrical studies of the neurone. Philadelphia: University of Pennsylvania Press.

Allen, C., \& Stevens, C. F. (1994). An evaluation of causes for unreliability of synaptic transmission. Proc. Natl. Acad. Sci. USA, 91, 10380-10383.

Barlow, H. B. (1972). Single units and sensation: A neuron doctrine for perceptual psychology? Perception, 1, 371-394. 
Bekkers, J. M., Richerson, G. B., \& Stevens, C. F. (1990). Origin of variability in quantal size in cultured hippocampal neurons and hippocampal slices. Proc. Natl. Acad. Sci. USA, 87(14), 5359-5362.

Bekkers, J. M., \& Stevens, C. F. (1994). The nature of quantal transmission at central excitatory synapses. Adv. Second Messenger Phosphoprotein Res., 29, 261-273.

Bekkers, J. M., \& Stevens, C. F. (1995). Quantal analysis of EPSCs recorded from small numbers of synapses in hippocampal cultures. J. Neurophysiol., 73, 11451156.

Bekkers, J. M., \& Stevens, C. F. (1996). Cable properties of cultured hippocampal neurons determined from sucrose-evoked miniature EPSCs. J. Neurophysiol., $75,1250-1255$.

Bialek, W., \& Rieke, F. (1992). Reliability and information-transmission in spiking neurons. Trends Neurosci., 15, 428-434.

Bialek, W., Rieke, F., van Steveninck, R. R. D., \& Warland, D. (1991). Reading a neural code. Science, 252, 1854-1857.

Britten, K. H., Shadlen, M. N., Newsome, W. T., \& Movshon, A. (1992). The analysis of visual motion: A comparison of neuronal and psychophysical performance. J. Neurosci., 12, 4745-4765.

Burnod, Y., \& Korn, H. (1989). Consequences of stochastic release of neurotransmitters for network computation in the central nervous system. Proc. Natl. Acad. Sci. USA, 86, 352-356.

Cover, T. M., \& Thomas, J. A. (1991). Elements of information theory. New York: Wiley.

DeFelice, L. J. (1981). Introduction to membrane noise. New York: Plenum Press.

DeWeese, M., \& Bialek, W. (1995). Information-flow in sensory neurons. Nuovo Cimento $D, 17,733-741$.

Dobrunz, L. E., Huang, E. P., \& Stevens, C. F. (1997). Very short-term plasticity in hippocampal synapses. Proc. Natl. Acad. Sci. USA, 94, 14843-14847.

Dobrunz, L. E., \& Stevens, C. F. (1997). Heterogeneity of release probability, facilitation, and depletion at central synapses. Neuron, 18, 995-1008.

Dobrunz, L. E., \& Stevens, C. F. (1999). Response of hippocampal synapses to natural stimulation patterns. Neuron, 22, 157-166.

Edwards, F. A., Konnerth, A., \& Sakmann, B. (1990). Quantal analysis of inhibitory synaptic transmission in the dendate gyrus of rat hippocampal slices: A patch-clamp study. J. Physiol., 430, 213-249.

Gabbiani, F. (1996). Coding of time-varying signals in spike trains of linear and half-wave rectifying neurons. Network: Comput. Neural Syst., 7, 61-85.

Gabbiani, F., \& Koch, C. (1996). Coding of time-varying signals in spike trains of integrate-and-fire neurons with random threshold. Neural Comput., 8, 4466.

Gabbiani, F., \& Koch, C. (1998). Principles of spike train analysis. In C. Koch \& I. Segev (Eds.), Methods in neuronal modeling: From ions to networks (2nd ed.). Cambridge, MA: MIT Press.

Green, D. M., \& Swets, J. A. (1966). Signal detection theory and psychophysics. New York: Wiley.

Helstrom, C. (1968). Statistical theory of signal detection. Oxford: Pergamon Press. 
Hessler, N. A., Shirke, A. M., \& Malinow, R. (1993). The probability of transmitter release at a mammalian central synapse. Nature, 366, 569-572.

Hille, B. 1992. Ionic channels of excitable membranes. Sunderland, MA: Sinauer Associates.

Katz, B. (1969). The release of neural transmitter substances. Liverpool: Liverpool University Press.

Koch, C. (1999). Biophysics of computation: Information processing in single neurons. New York: Oxford University Press.

Korn, H., \& Faber, D. S. (1991). Quantal analysis and synaptic efficacy in the CNS. Trends Neurosci., 14, 439-445.

Korn, H., \& Faber, D. S. (1993). Transmitter release mechanism: Relevance for neuronal network functions. In T. Poggio \& D. A. Glaser (Eds.), Exploring brain functions: Models in neuroscience (pp. 5-17). New York: Wiley.

Korn, H., Faber, D. S., \& Triller, A. (1986). Probabilistic determination of synaptic strength. J. Neurophysiol., 55, 402-421.

Larkman, A. U., Stratford, K., \& Jack, J. (1991). Quantal analysis of excitatory synaptic action and depression in hippocampal slices. Nature, 350, 344-347.

Laurent, G., \& Sivaramakrishnan, A. (1992). Single local interneurons in the locust make central synapses with different properties of transmitter release on distinct postsynaptic neurons. J. Neurosci., 12, 2370-2380.

Lisman, J. E. (1997). Bursts as a unit of neural information: Making unreliable synapses reliable. Trends Neurosci., 20, 38-43.

Manwani, A., \& Koch, C. (1998). Synaptic transmission: An informationtheoretic perspective. In M. Jordan, M. S. Kearns, \& S. A. Solla (Eds.), Advances in neural information processing systems, 10 (pp. 201-207). Cambridge, MA: MIT Press.

Manwani, A., \& Koch, C. (1999a). Detecting and estimating signals in noisy cable structures: I. Neuronal noise sources. Neural Comput., 11, 1797-1829.

Manwani, A., \& Koch, C. (1999b). Detecting and estimating signals in noisy cable structures: II. Information-theoretic analysis. Neural Comput., 11, 1831-1873.

Manwani, A., \& Koch, C. (1999c). Signal detection in noisy weakly-active dendrites. In M. S. Kearns, S. A. Solla, \& D. A. Cohn (Eds.), Advances in neural information processing systems, 11. Cambridge, MA: MIT Press.

Markram, H., \& Tsodyks, M. (1996). Redistribution of synaptic efficacy between neocortical pyramidal neurons. Nature, 382, 807-810.

Mason, A., Nicoll, A., \& Stratford, K. (1991). Synaptic transmission between individual pyramidal neurons of the rat visual cortex in vitro. J. Neurosci., 11, 72-84.

Moore, E. F., \& Shannon, E. E. (1956). Reliable circuits using less reliable relays. J. Franklin Inst., 262, 191-208.

Murthy, V. N., Sejnowski, T. J., \& Stevens, C. F. (1997). Heterogeneous release properties of visualized individual hippocampal synapses. Neuron, 18, 599612.

Newsome, W. T., Britten, K. H., \& Movshon, J. A. (1989). Neuronal correlates of a perceptual decision. Nature, 341, 52-54.

Papoulis, A. (1991). Probability, random variables, and stochastic processes. New York: McGraw-Hill. 
Perkel, D. H., \& Bullock, T. H. (1968). Neural coding. Neurosci. Res. Prog. Sum., 3, 405-527.

Poor, H. V. (1994). An introduction to signal detection and estimation. New York: Springer-Verlag.

Rall, W. (1967). Distinguishing theoretical synaptic potentials computed for different soma-dendritic distributions of synaptic input. J. Neurophysiol., 30, 1138-1168.

Redman, S. (1990). Quantal analysis of synaptic potentials in neurons of the central nervous system. Physiol. Rev., 70, 165-198.

Richmond, B. J., \& Optican, L. M. (1992). The structure and interpretation of neuronal codes in the visual system. In H. Wechsler (Ed.), Neural networks for perception (pp. 104-119). Orlando, FL: Academic Press.

Rieke, F., Warland, D., van Steveninck, R. D. R., \& Bialek, W. (1997). Spikes: Exploring the neural code. Cambridge, MA: MIT Press.

Rosenmund, C., Clements, J. D., \& Westbrook, G. L. (1993). Non-uniform probability of glutamate release at a hippocampal synapse. Science, 262, 754-757.

Shadlen, M. N., \& Newsome, W. T. (1998). The variable discharge of cortical neurons: Implications for connectivity, computation, and information coding. J. Neurosci., 18, 3870-3896.

Smetters, D. K., \& Zador, A. 1996. Synaptic transmission: Noisy synapses and noisy neurons. Curr. Biol., 6, 1217-1218.

Sorra, K. E., \& Harris, K. M. (1993). Occurrence and three-dimensional structure of multiple synapses between individual radiatum axons and their target pyramidal cells in hippocampal area CA1. J. Neurosci., 13, 3736-3748.

Steinmetz, P. N., Manwani, A., London, M., Segev, I., \& Koch, C. (2000). Subthreshold voltage noise due to channel fluctuations in active neuronal membranes. J. Comput. Neurosci.

Stevens, C. F. (1993). Quantal release of neurotransmitter and long-term potentiation. Neuron, 10, 55-64.

Stevens, C. F. (1994). Cooperativity of unreliable neurons. Curr. Biol., 4, 268-269.

Stevens, C. F., \& Wang, Y. (1994). Changes in reliability of synaptic function as a mechanism for plasticity. Nature, 371, 704-707.

Stevens, C. F., \& Wang, Y. (1995). Facilitation and depression at single central synapses. Neuron, 14, 795-802.

Stevens, C. F., \& Zador, A. (1996). Information through a spiking neuron. In D. S. Touretzky, M. C. Mozer, \& M. E. Hasselmo (Eds.), Advances in neural information processing systems, 8. Cambridge, MA: MIT Press.

Stratford, K. J., Tarczy-Hornoch, K., Martin, K. A., Bannister, N. J., \& Jack, J. J. (1996). Excitatory synaptic inputs to spiny stellate cells in cat visual cortex. Nature, 382, 258-261.

Strong, S. P., Koberle, R., van Steveninck, R. D. R., \& Bialek, W. 1998. Entropy and information in neural spike trains. Phys. Rev. Lett., 80, 197-200.

Traynelis, S. F., \& Jaramillo, F. (1998). Getting the most out of noise in the central nervous system. Trends Neurosci., 21, 137-145.

Tsodyks, M. V., \& Markram, H. (1997). The neural code between neocortical pyramidal neurons depends on neurotransmitter release probability. Proc. Natl. Acad. Sci. USA, 94, 719-723. 
von Neumann, J. (1956). The computer and the brain. New Haven, CT: Yale University Press.

Werner, G., \& Mountcastle, V. B. (1963). The variability of central neural activity in a sensory system and its implications for the central reflection of sensory events. J. Neurophysiol., 26, 958-977.

White, J. A., Rubinstein, J. T., \& Kay, A. R. (2000). Channel noise in neurons. Trends Neurosci., 23, 131-137.

Zador, A. (1998). Impact of synaptic unreliability on the information transmitted by spiking neurons. J. Neurophysiol., 79, 1219-1229.

Zador, A. M., \& Dobrunz, L. E. (1997). Dynamic synapses in the cortex. Neuron, 19, 1-4.

Zohary, E., Hillman, P., \& Hochstein, S. (1990). Time course of perceptual discrimination and single neuron reliability. Biol. Cybern., 62, 475-486.

Received September 22, 1999; accepted March 28, 2000. 